H. Saito and M. Yamauchi

Nagoya Math. J.

Vol. 76 (1979), 1-33

\title{
TRACE FORMULA OF CERTAIN HECKE OPERATORS FOR $\Gamma_{0}\left(q^{v}\right)$
}

\author{
HIROSHI SAITO AND MASATOSHI YAMAUCHI
}

\section{Introduction}

Let $S_{k}\left(\Gamma_{0}\left(q^{\nu}\right)\right)$ be the space of cusp forms of weight $\kappa$ with respect to the congruence subgroup $\Gamma_{0}\left(q^{\nu}\right)$, and $S_{k}^{0}\left(\Gamma_{0}\left(q^{\nu}\right)\right)$ its subspace of all new forms in $S_{n}\left(\Gamma_{0}\left(q^{\nu}\right)\right)$, where $q$ is a prime such that $q \geq 3$. Now for $f \in S_{\kappa}\left(\Gamma_{0}\left(q^{\nu}\right)\right)$, put $(f \mid W)(z)=f\left(\frac{-1}{q^{\nu} z}\right)\left(q^{\nu} z\right)^{x} q^{\nu \kappa, 2}$. Then it is known that $W$ induces an automorphism of $S_{x}\left(\Gamma_{0}\left(q^{\nu}\right)\right)$. On the other hand, for the character $\chi$ of $(Z / q Z)^{\times}$of order 2, let $\delta_{\chi}$ denote the "twisting operator" with respect to $\chi$, which was defined in [15] by Shimura, namely, $\left(f \mid \delta_{\chi}\right)(z)$ $=\sum_{n=1}^{\infty} a_{n} \chi(n) e^{2 \pi i n z}$ for $f(z)=\sum_{n=1}^{\infty} a_{n} e^{2 \pi i n z} S_{\kappa}\left(\Gamma_{0}\left(q^{\nu}\right)\right)$. If $\nu \geq 2, S_{\kappa}\left(\Gamma_{0}\left(q^{\nu}\right)\right)$ is closed under $\delta_{\chi}$ and if $\nu \geq 3$, using $W$ and $\delta_{x}$ we can decompose $S_{k}^{0}\left(\Gamma_{0}\left(q^{\nu}\right)\right)$ into four subspaces $S_{\mathrm{I}}, S_{\mathrm{II}}, S_{\mathrm{II}_{\chi}}$ and $S_{\mathrm{III}}$ in a natural way. For example, $S_{\mathrm{I}}$ is the space consisting of all $f \in S_{*}^{0}\left(\Gamma_{0}\left(q^{\nu}\right)\right)$ satisfying $f \mid W=f$ and $f \mid \delta_{x} W$ $=f \mid \delta_{\chi}$. We see these subspaces are closed under Hecke operators $T_{n}$ for all $n$. In the case where $\nu=2$, we can also consider such a decomposition of $S_{\kappa}^{0}\left(\Gamma_{0}\left(q^{\nu}\right)\right)$ and we shall discuss this case in $\S 5$. The main purpose of this paper is to give a formula for the trace of the Hecke operator $T_{n}$ on each subspace $S_{\mathrm{I}}, S_{\mathrm{II}}$, etc.. Noticing the trace $\operatorname{tr} T_{n}$ restricted on each subspace $S_{\mathrm{I}}, S_{\mathrm{II}}$, etc. can be expressed as a sum of $\operatorname{tr} T_{n}, \operatorname{tr} W T_{n}, \operatorname{tr} \delta_{x} W \delta_{x} T_{n}$, and $\operatorname{tr} \delta_{x} W \delta_{x} W T_{n}$ on $S_{\kappa}\left(\Gamma_{0}\left(q^{\nu}\right)\right)$, in $\S 2$ and $\S 3$, we shall give explicit formulae for $\operatorname{tr} \delta_{\chi} W \delta_{x} T_{n}$ and $\operatorname{tr} \delta_{x} W \delta_{x} W T_{n}$ on $S_{k}\left(\Gamma_{0}\left(q^{\nu}\right)\right)$ for $\nu \geq 2$ by means of Eichler-Selberg's trace formula and Hijikata's result [6] on the conjugacy with respect to $\Gamma_{0}(N)$. As an application of these formulae, we can give additions to the examples which were discussed by Doi and Yamauchi [4]. Namely, in this paper, we shall give several Fourier coefficients of a certain primitive cusp form $f \in S_{2}^{0}\left(\Gamma_{0}\left(q^{\nu}\right)\right)$ for $q^{\nu}=11^{3}$ and $q^{\nu}=19^{2}$, and

Received March 9, 1978.

During the preparation of this paper, the first-named author was partially supported by the Sonderforschungsbereich "Theoretische Mathematik", University of Bonn. 
discuss on the class field theoretical property of special points of the abelian variety associated with $f$. These examples turn out to be related to Shimura's theory on the construction of class fields over real quadratic fields. In $\S 6$, we shall give formulae for $\operatorname{tr} \delta_{\chi}$ and $\operatorname{tr} T_{q} W$ on $S_{x}\left(\Gamma_{0}\left(q^{\nu}\right), \psi\right)$. As a corollary of these formulae, we obtain another proof of the theorem of Asai ([2], Th. 4) and Ribet ([9], Prop. 4.4 and Th. 4.5) on the characterization of primitive cusp forms in $S_{\kappa}\left(\Gamma_{0}\left(q^{\nu}\right)\right)$ corresponding to $L$-functions of $\boldsymbol{Q}(\sqrt{-q})$.

\section{Notation}

The symbols $Z, Q, R$ and $C$ denote respectively the ring of rational integers, the rational number field, the real number field, and the complex number field. The symbol $\mathfrak{S}$ denotes the upper half complex plane:

$$
\mathfrak{S}=\{z \in C \mid \operatorname{Im} z>0\} \text {. }
$$

If we discuss a Fuchsian group of the first kind $\Gamma$ on $\mathfrak{S}$, then $\mathfrak{S C}^{*}$ denotes the union of $\mathfrak{F}$ and the cusps of $\Gamma$. For an associative ring $S$ with an identity element, we denote by $S^{\times}$the group of all invertible elements of $S$, and by $M_{n}(S)$ the ring of all square matrices of size $n$ with coefficients in $S$. Then we put $G L_{n}(S)=M_{n}(S)^{\times}$. For subsets $S_{i j}$ of $S, 1 \leq i, j \leq n$, $\left(S_{i j}\right)$ denotes the subset $\left\{\left(s_{i j}\right) \in M_{n}(S) \mid s_{i j} \in S_{i j}\right\}$. Let $G$ be a group and $H$ be its subgroup. We denote by $\tilde{H}$ the conjugacy with respect to $H$, i.e., $g \tilde{H} g^{\prime}$ if and only if $h^{-1} g h=g^{\prime}$ for $h \in H$. For a subset $X$ of $G$, let $X / \tilde{H}$ denote the quotient of $X$ by $\tilde{H}$ and sometimes also a complete system of representatives of $X / \tilde{H}$. For a finite-dimensional vector space $V$ over $C$ and a linear operator $T$ on $V, \operatorname{tr} T \mid V$ denotes the trace of $T$ on $V$.

\section{$\S 1$. Preliminaries}

In this section, we shall recall some facts on "twisting operator" defined in [15] and abelian varieties associated with primitive cusp forms, and make preliminary considerations. Let $\alpha=\left(\begin{array}{ll}a & b \\ c & d\end{array}\right) \in G L_{2}(R)$, $\operatorname{det} \alpha>0$. For a complex-valued function $f(z)$ on $\mathfrak{S}$ and a positive integer $\kappa \geq 2$, we define a function $f \mid[\alpha]_{\kappa}$ on $\mathfrak{S}$ by

$$
\left(f \mid[\alpha]_{k}\right)(z)=(\operatorname{det} \alpha)^{\kappa / 2}(c z+d)^{-\kappa} f(\alpha(z)),
$$

where $\alpha(z)=(a z+b) /(c z+d)$ for $z \in \mathfrak{F}$. For a positive integer $N$, put 


$$
\Gamma_{0}(N)=\left\{\left(\begin{array}{ll}
a & b \\
c & d
\end{array}\right) \in S L_{2}(Z) \mid c \equiv 0(\bmod N)\right\}
$$

For a character $\psi$ modulo $N$, we denote by $S_{\kappa}\left(\Gamma_{0}(N), \psi\right)$ the space of all holomorphic cusp forms on $\mathfrak{F}$ satisfying

$$
f \mid[\gamma]_{k}=\psi(a)^{-1} f \quad \text { for } \gamma=\left(\begin{array}{ll}
a & b \\
c & d
\end{array}\right) \in \Gamma_{0}(N),
$$

and by $S_{k}^{0}\left(\Gamma_{0}(N), \psi\right)$ the space of all new forms in $S_{k}\left(\Gamma_{0}(N), \psi\right)$. Throughout this paper, we fix a prime $q \geq 3$, and assume $N=q^{\nu}$ with a non-negative integer $\nu$. Let $\chi$ be a real primitive character of $(Z / q Z)^{\times}$of order 2 , and assume $\nu \geq 2$ and the conductor $\hat{f}_{\psi}$ of $\psi$ satisfies $\hat{f}_{\psi} \leq q^{\nu-1}$. Let $\alpha_{u}=\left(\begin{array}{ll}q & u \\ 0 & q\end{array}\right)$ for $u \in Z$, then as in [15] we can define the twisting operator $\delta_{x}$ on $S_{x}\left(\Gamma_{0}\left(q^{\nu}\right), \psi\right)$ with respect to $\chi$ by

$$
f\left|\delta_{\chi}=\frac{1}{W(\chi)} \sum_{u=1}^{q-1} \chi(u) f\right|\left[\alpha_{u}\right]_{\kappa}
$$

where $W(\chi)$ is the Gaussian sum for $\chi$. For $f \in S_{k}\left(\Gamma_{0}\left(q^{\nu}\right), \psi\right)$, put

$$
f|W=f|\left[\left(\begin{array}{rr}
0 & -1 \\
q^{\nu} & 0
\end{array}\right)\right]_{x}
$$

then $W$ gives an isomorphism of $S_{k}\left(\Gamma_{0}\left(q^{\nu}\right), \psi\right)$ onto $S_{k}\left(\Gamma_{0}\left(q^{\nu}\right), \bar{\psi}\right)$. In the following, except in $\S 6$, we assume $\psi$ is the identity character and $\kappa$ is an even positive integer. Put $S_{\kappa}\left(\Gamma_{0}\left(q^{\nu}\right)\right)=S_{\kappa}\left(\Gamma_{0}\left(q^{\nu}\right), \psi\right)$. By a result of AtkinLehner [3], we know $W$ induces an isomorphism of $S_{k}^{0}\left(\Gamma_{0}\left(q^{\nu}\right)\right)$ onto itself. On the other hand, if $\nu \geq 3$, we can prove

Proposition 1.1. If $\nu \geq 3, \delta_{x}$ induces an isomorphism

$$
\delta_{\chi}: S_{\kappa}^{0}\left(\Gamma_{0}\left(q^{\nu}\right)\right) \rightarrow S_{\kappa}^{0}\left(\Gamma_{0}\left(q^{\nu}\right)\right) .
$$

Proof. For a primitive form $f \in S_{\kappa}^{0}\left(\Gamma_{0}\left(q^{\nu}\right)\right)$, assume $f \mid \delta_{z}$ is contained in the space of old forms, then there exist $g_{1}, g_{2} \in S_{x}\left(\Gamma_{0}\left(q^{\nu-1}\right)\right)$ such that $\left(f \mid \delta_{x}\right)(z)$ $=g_{1}(z)+g_{2}(q z)$. Applying $\delta_{x}$ on both sides, we obtain $f\left|\delta_{x} \delta_{x}=g_{1}\right| \delta_{x}$. Since $\nu \geq 3, f \mid \delta_{x} \delta_{x}=f$ and $g_{1} \mid \delta_{x} \in S_{x}\left(\Gamma_{0}\left(q^{\nu-1}\right)\right)$. This contradicts our assumption.

If $\nu \geq 3$, by means of $\delta_{\alpha}$ and $W$, we can decompose $S_{x}^{0}\left(\Gamma_{0}\left(q^{\nu}\right)\right)$ into the following four spaces, namely,

$$
S_{\kappa}^{0}\left(\Gamma_{0}\left(q^{\nu}\right)\right)=S_{\mathrm{I}} \oplus S_{\mathrm{II}} \oplus S_{\mathrm{II}_{\chi}} \oplus S_{\mathrm{III}},
$$


where

$$
\begin{aligned}
S_{\mathrm{I}} & =\left\{f \in S_{x}^{0}\left(\Gamma_{0}\left(q^{\nu}\right)\right)|f| W=f, f\left|\delta_{\chi} W=f\right| \delta_{\chi}\right\} \\
S_{\mathrm{II}} & =\left\{f \in S_{x}^{0}\left(\Gamma_{0}\left(q^{\nu}\right)\right)|f| W=f, f\left|\delta_{\chi} W=-f\right| \delta_{\chi}\right\} \\
S_{\mathrm{II}} & =\left\{f \in S_{x}^{0}\left(\Gamma_{0}\left(q^{\nu}\right)\right)|f| W=-f, f\left|\delta_{\chi} W=f\right| \delta_{\chi}\right\} \\
S_{\mathrm{III}} & =\left\{f \in S_{k}^{0}\left(\Gamma_{0}\left(q^{\nu}\right)\right)|f| W=-f, f\left|\delta_{\chi} W=-f\right| \delta_{\chi}\right\} .
\end{aligned}
$$

Then we see easily that these four spaces are closed under Hecke operators, and that $S_{\mathrm{II}} \mid \delta_{x}=S_{\mathrm{II}}$. In the case where $\nu=2$, we can also consider a similar decomposition of $S_{x}^{0}\left(\Gamma_{0}\left(q^{2}\right)\right)$, however the situation is a little more complicated in this case, so we shall discuss it in detail in $\S 5$. To give a formula of traces of Hecke operators on the above spaces, let us consider the operators $\delta_{x} W \delta_{x}$ and $\delta_{x} W \delta_{x} W$ on $S_{k}\left(\Gamma_{0}\left(q^{\nu}\right)\right)$ for $\nu \geq 2$. These operators have a good property if $\nu \geq 3$, namely we can prove

Proposition 1.2. If $g$ is an old form in $S_{\kappa}\left(\Gamma_{0}\left(q^{\nu}\right)\right)$ with $\nu \geq 3$, then

$$
\begin{array}{r}
g \mid \delta_{\chi} W \delta_{\chi}=0 \\
g \mid \delta_{\chi} W \delta_{x} W=0 .
\end{array}
$$

Proof. If $g(z)=f(q z)$ for $f \in S_{x}\left(\Gamma_{0}\left(q^{\nu-1}\right)\right), g \mid \delta_{\chi}=0$. Hence we may assume $g$ is a primitive form with the level $q^{\lambda}, \lambda<\nu$. Then we see $\left(g \mid \delta_{\chi} W\right)(z)=f(q z)$ for some $f \in S_{\kappa}\left(\Gamma_{0}\left(q^{\nu-1}\right)\right)$, and we obtain $g \mid \delta_{\chi} W \delta_{\chi}=0$ and $g \mid \delta_{\chi} W \delta_{\chi} W=0$.

If $\nu \geq 3$, we can express the traces of Hecke operators on $S_{\mathrm{I}}, S_{\mathrm{II}}, S_{\mathrm{II} x}$, and $S_{\text {III }}$ by using $\operatorname{tr} T_{n}$, tr $W T_{n}, \operatorname{tr} \delta_{\chi} W \delta_{\chi} T_{n}$, and $\operatorname{tr} \delta_{\chi} W \delta_{\chi} W T_{n}$ on $S_{\kappa}\left(\Gamma_{0}\left(q^{\nu}\right)\right)$. For example, on $S_{\mathrm{I}}$, we have

$$
\begin{aligned}
\operatorname{tr} T_{n} \mid S_{\mathrm{I}}= & \frac{1}{4}\left\{\operatorname{tr} T_{n}\left|S_{\kappa}^{0}\left(\Gamma_{0}\left(q^{\nu}\right)\right)+\operatorname{tr} W T_{n}\right| S_{\kappa}^{0}\left(\Gamma_{0}\left(q^{\nu}\right)\right)\right. \\
& \left.+\operatorname{tr} \delta_{z} W \delta_{z} T_{n}\left|S_{x}\left(\Gamma_{0}\left(q^{\nu}\right)\right)+\operatorname{tr} \delta_{\chi} W \delta_{\chi} W T_{n}\right| S_{\kappa}\left(\Gamma_{0}\left(q^{\nu}\right)\right)\right\} .
\end{aligned}
$$

Formulae for $\operatorname{tr} T_{n}$ and $\operatorname{tr} W T_{n}$ are already given by Hijikata [6] and Yamauchi [16] respectively. In $\S 2$ and $\S 3$ we shall give formulae for $\operatorname{tr} \delta_{\chi} W \delta_{\chi} T_{n}$ and $\operatorname{tr} \delta_{z} W \delta_{x} W T_{n}$ on $S_{x}\left(\Gamma_{0}\left(q^{\nu}\right)\right)$ for $\nu \geq 2$ and for any $n$ prime to $q$. In the case where $\nu=2$, we can calculate the traces of Hecke operators on certain subspaces of $S_{n}\left(\Gamma_{0}\left(q^{2}\right)\right)$ by means of the above traces, we discuss it in $\S 5$.

In the rest of this section, we assume $\kappa=2$, and recall a few facts in [15]. Let $\Gamma$ be a group of level $q^{\nu}$ given by 


$$
\Gamma=\left\{\left(\begin{array}{ll}
a & b \\
c & d
\end{array}\right) \in \Gamma_{0}\left(q^{\nu}\right) \mid a \equiv d \equiv 1(\bmod q)\right\}
$$

Let $J$ be the jacobian variety of $\mathfrak{S}^{*} / \Gamma$ and $S_{2}(\Gamma)$ the vector space of all holomorphic cusp forms on $\mathscr{S}$ of weight 2 with respect to $\Gamma$. Then it is known that $J$ is defined over $\boldsymbol{Q}$. Let $f(z)=\sum_{n=1}^{\infty} a_{n} e^{2 \pi i n z}, a_{1}=1$, be a primitive cusp form in $S_{2}\left(\Gamma_{0}\left(q^{\nu}\right)\right.$ ), then $f$ is a common eigen-function for Hecke operators $T_{n}$ for all $n$ as an element of $S_{2}(\Gamma)$. Let $K$ be the subfield of $\boldsymbol{C}$ generated over $\boldsymbol{Q}$ by $a_{n}$ for all $n$. Then by [15, Th. 1], we have

(1.1) There exists a triple $(A, \mu, \theta)$ formed by the objects satisfying the following conditions.

(i) $A$ is a quotient of $J$ by an abelian variety defined over $\boldsymbol{Q}$, and $\mu$ is a natural map $J \rightarrow A$.

(ii) $\theta$ is an isomorphism of $K$ into End $(A) \otimes \boldsymbol{Q}$ such that $\mu \circ \xi_{n}$ $=\theta\left(a_{n}\right) \circ \mu$ for all $n . \quad\left(\xi_{n}\right.$ is an element of End $(J)$ associated with $T_{n}$.)

(iii) $\operatorname{dim} A=[K: Q]$.

Now if we take $N(=M)=q^{\nu}(\nu>1), r=q, s=N$ in the notation of [15, $\S 4]$, then we see our $\Gamma$ satisfies the condition (4.8) in $[15, \S 4]$. Suppose the following condition (the condition (4.9) in [15]) is satisfied:

(*) There is an automorphism $\rho$ of $K$, other than the identity map, such that $\chi(n) a_{n}=a_{n}^{o}$ for all $n$. (This implies especially that $\rho^{2}=1$ and $a_{n}=0$ if $(n, q) \neq 1$.)

Then by [15, Prop. 8 and Prop. 9], under the assumption (*), there exists an endomorphism $\eta$ of $A$ which is defined over the quadratic extension $k$ of $\boldsymbol{Q}$ corresponding to $\chi$ and satisfies the condition

(1.2) (i) $\eta^{s}=-\eta$ if $\varepsilon$ is the generator of $\mathrm{Gal}(k / \mathbf{Q})$.

(ii) $\eta^{2}=\chi(-1) q \cdot \mathrm{id}_{A}$.

(iii) $\eta \circ \theta(a)=\theta\left(a^{\rho}\right) \circ \eta$ for every $a \in K$.

Under the assumption $(*)$, we follow the procedure in [15]. Let $F$ be the invariant subfield of $K$ under $\rho$, and $\mathfrak{o}_{K}, \mathfrak{o}_{F}$ be the maximal orders of $K, F$ respectively. Let $\mathfrak{b}_{0}$ denote the ideal of $K$ generated by all $x$ in $\mathfrak{o}_{K}$ such that $x^{\rho}=-x$. We define the odd part $\mathfrak{b}$ of $\mathfrak{b}_{0}$ and an integral ideal $\mathfrak{c}$ of $\mathfrak{o}_{F}$ in exactly the same way as in $[14, \S 2]$ for the present $F$ and $K$. We put 


$$
\mathfrak{c}=\{t \in A \mid \theta(\mathfrak{b}) t=0\},
$$

then $\mathfrak{x}$ is isomorphic to $\left(\mathfrak{o}_{K} / \mathfrak{b}\right)^{2}$ as an $\mathfrak{o}_{K}$-module. Further we assume as in $[14, \S 9]$,

$(* *) \quad \chi(-1) q \equiv e^{2}(\bmod c)$ for some $e \in \mathfrak{o}_{F}$ prime to $\mathfrak{c}$,

and put

$$
\begin{aligned}
& \mathfrak{y}=\{t \in \mathfrak{x} \mid(\eta-\theta(e)) t=0\} \\
& \mathfrak{z}=\{t \in \mathfrak{x} \mid(\eta+\theta(e)) t=0\} .
\end{aligned}
$$

Then as in [14, Prop. 9.2], we can verify

(1.3) The submodules $\mathfrak{y}$ and $\mathfrak{z}$ are $\mathfrak{o}_{F}$-isomorphic to $\mathfrak{o}_{F} / \mathfrak{c}$, and $\mathfrak{x}=\mathfrak{y} \oplus \mathfrak{z}$.

Let $k(x)$ (resp. $k(\mathfrak{y}), k(\mathfrak{z})$ ) denote the smallest extension of $k$ over which the points of $\mathfrak{x}($ resp. $\mathfrak{y}, \mathfrak{z})$ are rational. Then $k(\mathfrak{x})$ is an abelian extension of $k$, and making $\mathrm{Gal}(k(\mathfrak{x}) / k)$ act on $\mathfrak{h}$ and $z$, we obtain an injective homomorphism

$$
\operatorname{Gal}(k(\mathfrak{c}) / k) \rightarrow\left(\mathfrak{o}_{F} / \mathfrak{c}\right)^{\times} \times\left(\mathfrak{o}_{F} / \mathfrak{c}\right)^{\times} .
$$

We shall discuss on the class-field theoretical properties of $\left(\mathfrak{o}_{F} / \mathfrak{c}\right)$-valued "character" associated with the abelian extensions $k(\mathfrak{y})$ and $k(\mathfrak{z})$ for some numerical examples in $\S 4$ and $\S 5$.

\section{§2. A formula for $\operatorname{tr} \delta_{\chi} W \delta_{\chi} W T_{n}$}

For a rational prime $p$, let $\boldsymbol{Z}_{p}$ and $\boldsymbol{Q}_{p}$ denote the ring of $p$-adic integers and the field of $p$-adic numbers respectively. Let $\boldsymbol{Q}_{A}$ (resp. $\boldsymbol{Q}_{A}^{\times}$) be the adele ring (resp. the idele group) of $\boldsymbol{Q}$. For a non-negative integer $\mu$ and a prime $p$, put

$$
R_{p}(\mu)=\left(\begin{array}{ll}
Z_{p} & Z_{p} \\
p^{\mu} Z_{p} & Z_{p}
\end{array}\right)
$$

For a prime $p$ different from $q$ (resp. $p=q$ ), let $U_{p}$ denote $R_{p}(0)^{\times}=G L_{2}\left(Z_{p}\right)$ (resp. $\left.R_{q}(\nu)^{\times}\right)$and let $U_{\infty}$ denote the subgroup $\left\{g \in G L_{2}(R) \mid \operatorname{det} g>0\right\}$ of $G L_{2}(R)$. Then $U=\prod_{p} U_{p} \times U_{\infty}$ is an open subgroup of $G L_{2}\left(\boldsymbol{Q}_{A}\right)$, and $\Gamma_{0}\left(q^{\nu}\right)$ $=U \cap G L_{2}(\boldsymbol{Q})$.

Lemma 2.1. The notation being as above, one has

$$
\left(\prod_{p \neq q} U_{p} \times U_{q}\left(\begin{array}{ll}
q & 1 \\
0 & q
\end{array}\right) U_{q} \times U_{\infty}\right) \cap G L_{2}(\boldsymbol{Q})=\bigcup_{i=1}^{q-1} \Gamma_{0}\left(q^{\nu}\right)\left(\begin{array}{ll}
q & i \\
0 & q
\end{array}\right),
$$


and the right hand side is a disjoint union.

Proof. It is easy to see that the right hand side is contained in the left hand side and that the union is disjoint. Let $g=\left(\begin{array}{ll}a & b \\ c & d\end{array}\right)$ be an element contained in the left hand side, then $\operatorname{det} g=q^{2}$. If $c \neq 0$, there exist $a^{\prime}, c^{\prime} \in Z$ prime to $q$ such that $a=q a^{\prime} c=q^{\mu} c^{\prime}$ with a positive integer $\mu \geq \nu+1$ and $\left(a^{\prime}, c^{\prime}\right)=1$. Hence there exists an element $h=\left(\begin{array}{cc}m & n \\ -q^{\mu-1} c^{\prime} & a^{\prime}\end{array}\right)$ $\in \Gamma_{0}\left(q^{\nu}\right)$ for some $m, n \in Z$. Since $h g=\left(\begin{array}{ll}* & * \\ 0 & *\end{array}\right)$ and $\operatorname{det} g=q^{2}$, we may assume $c=0$ and $g$ is of the form $\left(\begin{array}{ll}q & b \\ 0 & q\end{array}\right)$. Now we easily see such elements are contained in the right hand side. This completes the proof of our lemma.

For $g=\left(\begin{array}{ll}a & b \\ c & d\end{array}\right) \in\left(\prod_{p \neq q} U_{p} \times U_{q}\left(\begin{array}{ll}q & 1 \\ 0 & q\end{array}\right) U_{q} \times U_{\infty}\right) \cap G L_{2}(\boldsymbol{Q})$, we put

$$
\chi_{0}(g)=\chi(a / q) \chi(b) \text {, }
$$

then we can check

$$
\chi_{0}\left(\gamma g \gamma^{\prime}\right)=\chi_{0}(g) \quad \text { for } \gamma, \gamma^{\prime} \in \Gamma_{0}\left(q^{\nu}\right) .
$$

Let $\left(\prod_{p \neq q} U_{p} \times U_{q}\left(\begin{array}{ll}q & 1 \\ 0 & q\end{array}\right) U_{q} \times U_{\infty}\right) \cap G L_{2}(\boldsymbol{Q})=\bigcup_{\lambda=1}^{q-1} \Gamma_{0}\left(q^{\nu}\right) \alpha_{\lambda}$ be a disjoint union, then we have for $f \in S_{k}\left(\Gamma_{0}\left(q^{\nu}\right)\right)$,

$$
f\left|\delta_{\chi}=\frac{1}{W(\chi)} \sum_{\lambda=1}^{q-1} \chi_{0}\left(\alpha_{\lambda}\right) f\right|\left[\alpha_{\lambda}\right]_{\kappa} .
$$

For $\nu \geq 2$, let $\Xi_{q}\left(\delta_{z} W \delta_{x} W\right)$ denote the subset of $R_{q}(\nu)$ given by

$$
\Xi_{q}\left(\delta_{x} W \delta_{\chi} W\right)=\left\{\begin{array}{l}
\left(\begin{array}{ll}
q^{\nu+2} Z_{q}^{\times} & q^{\nu+1} Z_{q}^{\times} \\
q^{2 \nu+1} Z_{q}^{\times} & q^{\nu+2} Z_{q}^{\times}
\end{array}\right), \quad \nu \geq 3 \\
\left\{g \in\left(\begin{array}{ll}
q^{4} Z_{q}^{\times} & q^{3} Z_{q}^{\times} \\
q^{5} Z_{q}^{\times} & q^{4} Z_{q}^{\times}
\end{array}\right) \mid v_{q}(\operatorname{det} g)=8\right\}, \quad \nu=2,
\end{array}\right.
$$

where $v_{q}$ denotes the valuation of $Z_{q}$ such that $v_{q}(q)=1$. Then $\Xi_{q}\left(\delta_{\chi} W \delta_{\chi} W\right)$ is a union of $U_{q}$-double cosets. For $i, j, 1 \leq i, j \leq q-1$, put

$$
\begin{aligned}
\alpha_{i j} & =\left(\begin{array}{ll}
q & i \\
0 & q
\end{array}\right)\left(\begin{array}{rr}
0 & -1 \\
q^{\nu} & 0
\end{array}\right)\left(\begin{array}{ll}
q & j \\
0 & q
\end{array}\right)\left(\begin{array}{rr}
0 & -1 \\
q^{\nu} & 0
\end{array}\right) \\
& =\left(\begin{array}{cc}
i j q^{2 \nu}-q^{\nu+2} & -i q^{\nu+1} \\
j q^{2 \nu+1} & -q^{\nu+2}
\end{array}\right) .
\end{aligned}
$$


LEMMA 2.2. Assume $\nu \geq 2$, and put $\alpha_{i j}=\left(\begin{array}{cc}i j q^{2 \nu}-q^{\nu+2} & -i q^{\nu+1} \\ j q^{2 \nu+1} & -q^{\nu+2}\end{array}\right)$ for $i, j, 1 \leq i, j \leq q-1$. Then one has

$$
\Xi_{q}\left(\delta_{x} W \delta_{x} W\right)=\bigcup_{i, j=1}^{q-1} U_{q} \alpha_{i j}
$$

and the right hand side is a disjoint union.

Proof. The right hand side is clearly contained in the left hand side. For $i, j, i^{\prime}, j^{\prime}, 1 \leq i, j, i^{\prime}, j^{\prime} \leq q-1$, we have

$$
\alpha_{i j} \alpha_{i^{\prime} j^{\prime}}^{-1}=\frac{1}{q^{4}}\left(\begin{array}{cc}
q^{4}-i q^{\nu+2}\left(j-j^{\prime}\right) & q^{3}\left(i-i^{\prime}\right)+i i^{\prime} q^{\nu+1}\left(j-j^{\prime}\right) \\
-q^{\nu+3}\left(j-j^{\prime}\right) & i^{\prime} q^{\nu+2}\left(j-j^{\prime}\right)+q^{4}
\end{array}\right),
$$

and the disjointness of the right hand side follows from this easily. Hence to prove our lemma, it is enough to show $\Xi_{q}\left(\delta_{z} W \delta_{z} W\right) \subset \bigcup_{i, j=1}^{q-1} U_{q} \alpha_{i j}$. Let $\alpha$ be an element of $\Xi_{q}\left(\delta_{x} W \delta_{z} W\right)$. We may assume $\alpha=\left(\begin{array}{ll}q^{\nu+2} a & q^{\nu+1} \\ q^{2 \nu+1} c & q^{\nu+2}\end{array}\right)$ with $a, c \in Z_{q}$. For $\alpha=\left(\begin{array}{ll}q^{\nu+2} a & q^{\nu+1} \\ q^{2 \nu+1} c & q^{\nu+2}\end{array}\right)$ and $\alpha^{\prime}=\left(\begin{array}{ll}q^{\nu+2} a^{\prime} & q^{\nu+1} \\ q^{2 \nu+1} c^{\prime} & q^{\nu+2}\end{array}\right)$, by a direct calculation we can verify easily

$$
U_{q} \alpha=U_{q} \alpha^{\prime} \quad \text { if and only if } a \equiv a^{\prime}, c \equiv c^{\prime}(\bmod q) .
$$

Since $U_{q} \alpha_{i j}=U_{q}\left(\begin{array}{cc}-j q^{2 \nu}+i^{-1} q^{\nu+2} & q^{\nu+1} \\ -j q^{2 \nu+1} & q^{\nu+2}\end{array}\right)$, we obtain $\Xi_{q}\left(\delta_{\chi} W \delta_{\chi} W\right)=\bigcup_{i, j=1}^{q-1} U_{q} \alpha_{i j}$.

Let $n$ be a positive integer with $(n, q)=1$. For a prime $p \neq q$, we put

$$
\Xi_{p}(n)=\left\{g \in M_{2}\left(Z_{p}\right) \mid v_{p}(\operatorname{det} g)=v_{p}(n)\right\},
$$

where $v_{p}$ is the valuation of $Z_{p}$ given by $v_{p}(p)=1$. Then $\Xi_{p}(n)$ is a union of $U_{p}$-double cosets.

LEMMA 2.3. The notation being as above, let $n$ be a positive integer prime to $q$, and assume $\nu \geq 2$. If $\left(\prod_{p \neq q} \Xi_{p}(n) \times U_{q} \times U_{\infty}\right) \cap G L_{2}(Q)$ $=\bigcup_{\lambda=1}^{d} \Gamma_{0}\left(q^{\nu}\right) \beta_{\lambda}$ is a disjoint union, then one has

$$
\begin{gathered}
\left(\prod_{p \neq q} \Xi_{p}(n) \times \Xi_{q}\left(\delta_{\chi} W \delta_{\chi} W\right) \times U_{\infty}\right) \cap G L_{2}(\boldsymbol{Q}) \\
=\bigcup_{i, j=1}^{q-1} \bigcup_{\lambda=1}^{d} \Gamma_{0}\left(q^{\nu}\right) \alpha_{i j} \beta_{\lambda},
\end{gathered}
$$

and the right hand side is a disjoint union. 
Proof. Since $\prod_{p \neq q} \Xi_{p}(n) \times U_{\infty}=\bigcup_{k=1}^{d}\left(\prod_{p \neq q} U_{p} \times U_{\infty}\right) \beta_{2}$ is a disjoint union, we see by Lemma 2.2

$$
\prod_{p \neq q} \Xi_{p}(n) \times \Xi_{q}\left(\delta_{\chi} W \delta_{\chi} W\right) \times U_{\infty}=\bigcup_{i, j=1}^{q-1} \bigcup_{\lambda=1}^{d} U \alpha_{i j} \beta_{\lambda},
$$

and the right hand side is a disjoint union. By considering the intersections of both sides with $G L_{2}(\boldsymbol{Q})$, we obtain our assertion.

For $\alpha_{q}=\left(\begin{array}{ll}a & b \\ c & d\end{array}\right) \in \Xi_{q}\left(\delta_{x} W \delta_{x} W\right)$, we put

$$
\chi_{1}\left(\alpha_{q}\right)=\chi\left(-b c / q^{3 \nu+2}\right) .
$$

Then for $\gamma_{q} \in U_{q}$, we have

$$
\chi_{1}\left(\gamma_{q}^{-1} \alpha_{q} \gamma_{q}\right)=\chi_{1}\left(\alpha_{q}\right) .
$$

For $\alpha \in \prod_{p \neq q} \Xi_{p}(n) \times \Xi_{q}\left(\delta_{x} W \delta_{x} W\right) \times U_{\infty}$, we define $\chi_{1}(\alpha)$ by

$$
\chi_{1}(\alpha)=\chi_{1}\left(\alpha_{q}\right)
$$

where $\alpha_{q}$ denotes the $q$-component of $\alpha$. Then we can verify

$$
\chi_{1}\left(\gamma \alpha \gamma^{\prime}\right)=\chi_{1}(\alpha) \quad \text { for } \gamma, \gamma^{\prime} \in \Gamma_{0}\left(q^{\nu}\right) \text {. }
$$

For a positive integer $n$ prime to $q$, put

$$
\Xi\left(\delta_{\chi} W \delta_{\chi} W T_{n}\right)=\left(\prod_{p \neq q} \Xi_{p}(n) \times \Xi_{q}\left(\delta_{\chi} W \delta_{\chi} W\right) \times U_{\infty}\right) \cap G L_{2}(\mathbf{Q}),
$$

then $\Xi\left(\delta_{z} W \delta_{z} W T_{n}\right)$ is a union of $\Gamma_{0}\left(q^{\nu}\right)$-double cosets. If we put for $f \in S_{x}\left(\Gamma_{0}\left(q^{\nu}\right)\right)$

$$
\left.f\right|_{\chi} \Xi\left(\delta_{\chi} W \delta_{\chi} W T_{n}\right)=\frac{n^{\alpha / 2-1} \chi(n)}{W(\chi)^{2}} \sum_{\alpha \in \Gamma_{0}\left(q^{*}\right) \backslash \xi\left(\delta_{\chi} W \delta_{x} W T_{n}\right)} \chi_{1}(\alpha) f \mid[\alpha]_{k},
$$

then by (2.3) the right hand side is independent of the choice of the representatives $\alpha$ 's, and by Lemma 2.3 , we obtain

$$
f\left|\delta_{\chi} W \delta_{\chi} W T_{n}=f\right|_{\chi} \Xi\left(\delta_{\chi} W \delta_{\chi} W T_{n}\right) .
$$

By Eichler-Selberg's trace formula ([5], [7], [10], [11]), we can express $\operatorname{tr} \delta_{\chi} W \delta_{\chi} W T_{n} \mid S_{x}\left(\Gamma_{0}\left(q^{\nu}\right)\right)$ as a sum extended over some conjugacy classes with respect to $\Gamma_{0}\left(q^{\nu}\right)$. By noticing $\Xi\left(\delta_{x} W \delta_{x} W T_{n}\right)=\left(\begin{array}{rr}-1 & 0 \\ 0 & 1\end{array}\right) \Xi\left(\delta_{x} W \delta_{x} W T_{n}\right)\left(\begin{array}{rr}-1 & 0 \\ 0 & 1\end{array}\right)^{-1}$ and $\chi_{1}\left(\left(\begin{array}{ll}0 & 1 \\ 1 & 0\end{array}\right) \alpha\left(\begin{array}{ll}0 & 1 \\ 1 & 0\end{array}\right)^{-1}\right)=\chi_{1}(\alpha)$ for $\alpha \in \Xi\left(\delta_{x} W \delta_{z} W T_{n}\right)$, we obtain 


$$
\begin{aligned}
& \operatorname{tr} \delta_{\chi} W \delta_{\chi} W T_{n} \mid S_{\kappa}\left(\Gamma_{0}\left(q^{\nu}\right)\right)=\frac{\chi(n)}{W(\chi)^{2}}\left(t_{e}+t_{h}+t_{p}\right) \\
& t_{e}=-\frac{1}{4} \sum_{\alpha \in B\left(\delta_{\chi} W \delta_{\chi} W T_{n}\right)_{e} / \Gamma_{0}\left(q^{\nu}\right)} \chi_{1}(\alpha) \frac{1}{[\Gamma(\alpha):\{ \pm 1\}]} \frac{\zeta_{\alpha}^{\alpha-1}-\eta_{\alpha}^{\kappa-1}}{\zeta_{\alpha}-\eta_{\alpha}} q^{-(2 \nu+4)(\alpha / 2-1)} \\
& t_{h}=-\frac{1}{2} \sum_{\alpha \in \delta\left(\delta_{x} W \delta_{x} W T_{n}\right)_{h} / \Gamma_{0}\left(q^{\nu}\right)} \chi_{1}(\alpha) \frac{\left(\min .\left(\left|\zeta_{\alpha}\right|,\left|\eta_{\alpha}\right|\right)\right)^{\kappa-1}}{\left|\zeta_{\alpha}-\eta_{\alpha}\right|} q^{-(2 \nu+4)(\kappa / 2-1)} \\
& t_{p}=-\lim _{s \rightarrow 0} \frac{s}{8} \sum_{\alpha \in \delta\left(\delta_{x} W \delta_{x} W T_{n}\right)_{p} / \Gamma_{0}\left(q^{\nu}\right)} \chi_{1}(\alpha) \frac{1}{|m(\alpha)|^{1+s}} n^{\kappa / 2-1}
\end{aligned}
$$

where $\Xi\left(\delta_{x} W \delta_{x} W T_{n}\right)_{e}$ (resp. $\Xi\left(\delta_{x} W \delta_{z} W T_{n}\right)_{h}$; resp. $\left.\Xi\left(\delta_{x} W \delta_{x} W T_{n}\right)_{p}\right)$ denotes the set of all elliptic elements (resp. hyperbolic elements fixing cusps of $\Gamma_{0}\left(q^{\nu}\right)$; resp. parabolic elements fixing cusps of $\left.\Gamma_{0}\left(q^{\nu}\right)\right)$ in $\Xi\left(\delta_{z} W \delta_{z} W T_{n}\right)$. For $\alpha \in G L_{2}(Q), \zeta_{\alpha}$ and $\eta_{\alpha}$ are the two characteristic roots of $\alpha$ and $\Gamma(\alpha)$ $=\left\{\gamma \in \Gamma_{0}\left(q^{\nu}\right) \mid \gamma^{-1} \alpha \gamma=\alpha\right\}$. For a parabolic element $\alpha$ in $G L_{2}(Q) \cap U_{\infty}$ fixing a cusp of $\Gamma_{0}\left(q^{\nu}\right)$, there exists $g \in G L_{2}(Q) \cap U_{\infty}$ such that $g \Gamma(\alpha) g^{-1}$ $=\left\{\left(\begin{array}{cc}1 & m h \\ 0 & 1\end{array}\right) \mid m \in Z\right\}$ and $g \alpha g^{-1}=\left(\begin{array}{cc}\zeta_{\alpha} & \lambda_{\alpha} \\ 0 & \zeta_{\alpha}\end{array}\right)$ with $h>0$, then $m(\alpha)=\lambda_{\alpha} \zeta_{\alpha}^{-1} / h$.

To express $\operatorname{tr} \delta_{x} W \delta_{x} W T_{n}$ in a more explicit form, we follow Hijikata [6]. First let us introduce some notation. For a quadratic polynomial $\Phi(X)=X^{2}-s X+n$ in $Z[X]$, we put

$$
K(\Phi)=\boldsymbol{Q}[X] /(\Phi(X)),
$$

then $K(\Phi)$ is a commutative $\boldsymbol{Q}$-algebra of dimension 2 over $\boldsymbol{Q}$. We denote by $\tilde{X}$ the class of $X$ in $K(\Phi)$. By a $Z$-order of $K(\Phi)$ we understand a subring $\Lambda$ of $K(\Phi)$ containing the unity which is a free $Z$-module of rank 2. For a prime $p$, we put $K(\Phi)_{p}=K(\Phi) \otimes_{Q} \boldsymbol{Q}_{p}$ and $\Lambda_{p}=\Lambda \otimes_{Z} Z_{p}$. Let $\alpha$ be an element of $G L_{2}(Q)$ with the minimal polynomial $\Phi(X)$. For a $Z$ order $\Lambda$ containing $\tilde{X}$ and a non-negative integer $\mu$, put

$$
C_{p}(\mu, \alpha, \Lambda)=\left\{g^{-1} \alpha g \mid g \in G L_{2}\left(\boldsymbol{Q}_{p}\right), \varphi\left(\Lambda_{p}\right)=g R_{p}(\mu) g^{-1} \cap \boldsymbol{Q}_{p}[\alpha]\right\},
$$

where $Q_{p}[\alpha]$ is a $\boldsymbol{Q}_{p}$-algebra generated by $\alpha$ and $\varphi$ is a canonical isomorphism of $K(\Phi)_{p}$ to $\boldsymbol{Q}_{r}[\alpha]$ such that $\varphi(\tilde{X})=\alpha$. For the infinite prime, we put

$$
C_{\infty}(\alpha, \Lambda)=\left\{g^{-1} \alpha g \mid g \in G L_{2}(R)\right\} .
$$

Theorem 2.4 (Hijikata [6]). The notation being as above, let $\rho$ be a non-negative integer such that $\left[\Lambda_{p}: Z_{p}[\tilde{X}]\right]=p^{\rho}$. For a non-negative integer $\mu$, put 


$$
\begin{aligned}
& \Omega_{p}(\mu, \Phi, \Lambda)=\left\{\xi \in Z_{p} \mid \Phi(\xi) \equiv 0\left(\bmod p^{\mu+2 \rho}\right)\right\} \\
& \Omega_{p}^{\prime}(\mu, \Phi, \Lambda)=\left\{\begin{array}{c}
\left\{\eta \in Z_{p} \mid \Phi(\eta) \equiv 0\left(\bmod p^{\mu+2 \rho+1}\right)\right\} \\
\quad \text { if } p^{-2 \rho}\left(s^{2}-4 n\right) \equiv 0(\bmod p) \text { and } \mu>0 \\
\phi, \text { otherwise, }
\end{array}\right.
\end{aligned}
$$

and let $\Omega$ and $\Omega^{\prime}$ be a complete system of representatives of $\Omega_{p}(\mu, \Phi, \Lambda)$ modulo $p^{\mu+\rho}$ and $\Omega_{p}^{\prime}(\mu, \Phi, \Lambda)$ modulo $p^{\mu+\rho}$ respectively. For $\xi \in \Omega_{p}(\mu, \Phi, \Lambda)$, $\eta \in \Omega_{p}^{\prime}(\mu, \Phi, \Lambda)$, put

$$
\begin{aligned}
\varphi_{\xi}(\tilde{X}) & =\left(\begin{array}{cc}
\xi & p^{\rho} \\
-p^{-\rho} \Phi(\xi) & s-\xi
\end{array}\right) \\
\varphi_{\prime \prime}^{\prime}(\tilde{X}) & =\left(\begin{array}{cc}
s-\eta & -p^{-\mu-\rho} \Phi(\eta) \\
p^{\mu+\rho} & \eta
\end{array}\right) .
\end{aligned}
$$

Then $\left\{\varphi_{\xi}(\tilde{X}) \mid \xi \in \Omega\right\} \cup\left\{\varphi_{\eta}^{\prime}(\tilde{X}) \mid \eta \in \Omega^{\prime}\right\}$ gives a complete system of representatives of $C_{p}(\mu, \Phi, \Lambda) / \widetilde{R_{p}(\mu)^{\times}}$.

CoRollary 2.5. The notation being as above, one has

$$
\left|C_{p}(0, \alpha, \Lambda) / \widetilde{R_{p}(0)} \times\right|=1 .
$$

These are the special cases of Th. 2.3 and its corollary of [6].

We put $C_{p}(\alpha, \Lambda)=C_{p}(0, \alpha, \Lambda)$ if $p \neq q$ and $C_{q}(\alpha, \Lambda)=C_{q}(\nu, \alpha, \Lambda)$. Let $R\left(q^{\nu}\right)$ be a $Z$-order of $M_{2}(Q)$ such that $R\left(q^{\nu}\right) \otimes_{Z} Z_{p}=R_{p}(0)$ for $p \neq q$ and $R\left(q^{\nu}\right) \otimes_{Z} Z_{q}=R_{q}(\nu)$. For a $Z$-order $\Lambda$ of $K(\Phi)$ containing $\tilde{X}$, put

$$
\begin{aligned}
C(\alpha, \Lambda) & =\left\{g^{-1} \alpha g \mid g \in G L_{2}(\boldsymbol{Q}), \varphi(\Lambda)=g R\left(q^{\nu}\right) g^{-1} \cap \boldsymbol{Q}[\alpha]\right\} \\
C_{A}(\alpha, \Lambda) & =\left\{g^{-1} \alpha g \mid g \in G L_{2}\left(\boldsymbol{Q}_{A}\right), g_{p} \in C_{p}(\alpha, \Lambda)\right\}
\end{aligned}
$$

where $g_{p}$ denotes the $p$-component of $g$, and define the class number of $\Lambda$ by

$$
h(K(\Phi), \Lambda)=\left|\left(K(\Phi) \otimes_{Q} \boldsymbol{Q}_{A}\right)^{\times} / K(\Phi)^{\times}\left(\prod_{p} \Lambda_{p}^{\times} \times \Lambda_{\infty}^{+}\right)\right| .
$$

Here $\Lambda_{\infty}^{+}$denotes the subgroup of $\left(K(\Phi) \otimes_{Q} R\right)^{\times}$consisting of all elements with positive determinants for the regular representation of $\left(K(\Phi) \otimes_{Q} R\right)$.

Proposition 2.6. Let $\alpha$ be an element of $G L_{2}(Q)$ with the minimal polynomial $\Phi(X)=X^{2}-s X+n \in Z[X]$. Let $\theta$ be the canonical map from $C(\alpha, \Lambda) / \widetilde{\Gamma_{0}\left(q^{\nu}\right)}$ to $C_{A}(\alpha, \Lambda) / \tilde{U}$ induced by the inclusion $C(\alpha, \Lambda) \subset C_{A}(\alpha, \Lambda)$. For each prime $p$, let $\Xi_{p}$ be a union of $U_{p}$-double cosets and assume $\Xi_{p}$ 
$=U_{p}$ except a finite number of primes. Put $\Xi_{A}=\prod_{p} E_{p} \times U_{\infty}$ and $E$ $=\Xi_{A} \cap G L_{2}(Q)$, then one has

i) $\theta$ induces a surjective map

$$
\theta: C(\alpha, \Lambda) \cap \Xi / \widetilde{\Gamma_{0}\left(q^{\nu}\right)} \rightarrow C_{A}(\alpha, \Lambda) \cap \Xi_{A} / \tilde{U}
$$

ii) For each class $\tilde{\alpha}$ in $C_{A}(\alpha, \Lambda) \cap \Xi_{A} / \tilde{U}$,

$$
\left|\theta^{-1}(\tilde{\alpha})\right|=h(K(\Phi), \Lambda) .
$$

iii) $\left|C_{A}(\alpha, \Lambda) \cap \Xi_{A}\right| \tilde{U}|=| \prod_{p} C_{p}(\alpha, \Lambda) \cap \Xi_{p}\left|\tilde{U}_{p}\right| \times\left|C_{\infty}(\alpha, \Lambda) \cap U_{\infty}\right| \tilde{U}_{\infty} \mid$.

Proof. Let $g^{-1} \alpha g$ be an element of $C_{A}(\alpha, \Lambda) \cap \Xi_{A}$, then there exist $g_{1} \in G L_{2}(\boldsymbol{Q})$ and $g_{2} \in U$ such that $g=g_{1} g_{2}$, since $G L_{2}\left(\boldsymbol{Q}_{A}\right)=G L_{2}(\boldsymbol{Q}) U$. We see $g^{-1} \alpha g \tilde{U} g_{1}^{-1} \alpha g_{1}$ and $g_{1}^{-1} \alpha g_{1} \in C(\alpha, \Lambda) \cap \Xi$, and i) follows from this. Let $g \in G L_{2}\left(\boldsymbol{Q}_{A}\right)$ be an element such that $g^{-1} \alpha g$ is contained in $\tilde{\alpha}$. Then we see $\theta^{-1}(\tilde{\alpha})$ is in one to one correspondence with $\boldsymbol{Q}[\alpha]^{\times} \backslash\left(\left(\boldsymbol{Q}[\alpha] \otimes_{\boldsymbol{Q}} \boldsymbol{Q}_{A}\right)^{\times} g U\right)$ $\cap G L_{2}(Q) / \Gamma_{0}\left(q^{\nu}\right)$. Since $g_{1}^{-1} \alpha g_{1} \in C(\alpha, \Lambda) \cap \Xi$, we have

$$
\begin{aligned}
\mid \boldsymbol{Q}[\alpha]^{\times} \backslash & \left.\left(\boldsymbol{Q}[\alpha] \otimes_{\boldsymbol{Q}} \boldsymbol{Q}_{A}\right)^{\times} g U\right) \cap G L_{2}(\boldsymbol{Q})\left|\Gamma_{0}\left(q^{\nu}\right)\right| \\
& =\left|Q[\alpha]^{\times} \backslash\left(\boldsymbol{Q}[\alpha] \otimes_{\boldsymbol{Q}} \boldsymbol{Q}_{A}\right)^{\times} g U g^{-1} / g U g^{-1}\right| \\
& =\left|\boldsymbol{Q}[\alpha]^{\times} \backslash\left(\boldsymbol{Q}[\alpha] \otimes_{\boldsymbol{Q}} \boldsymbol{Q}_{A}\right)^{\times} /\left(\boldsymbol{Q}[\alpha] \otimes_{\boldsymbol{Q}} \boldsymbol{Q}_{A}\right)^{\times} \cap g U g^{-1}\right| \\
& =h(K(\Phi), \Lambda),
\end{aligned}
$$

thus we have proved the assertion ii). If $p \neq q$ and $\Xi_{p}=U_{p}$, by Cor. 2.4, we have $\left|C_{p}(\alpha, \Lambda) \cap \Xi_{p}\right| \tilde{U}_{p} \mid=1$, and iii) follows from this easily.

Now we are ready to give a formula for $\operatorname{tr} \delta_{x} W \delta_{x} W T_{n} \mid S_{k}\left(\Gamma_{0}\left(q^{\nu}\right)\right)$.

TheOREM 2.7. Let $n$ be a positive integer prime to $q$ and $\kappa$ be an even positive integer. Assume $\nu \geq 2$, then one has

$$
\operatorname{tr} \delta_{z} W \delta_{x} W T_{n} \mid S_{x}\left(\Gamma_{0}\left(q^{\nu}\right)\right)=\frac{\chi(n)}{W(\chi)^{2}}\left(t_{e}+t_{h}+t_{p}\right),
$$

where

$$
\begin{aligned}
& t_{e}=-\frac{1}{2} \sum_{s^{2}-4 n<0} \frac{\zeta^{k-1}-\eta^{k-1}}{\zeta-\eta} c_{q}\left(\Phi_{s}\right) \sum_{\substack{\left.\left.f^{2} \mid l s^{2}-4 n\right) \\
\left(s^{2}-4 n\right) / f^{2}=1\right)=1 \\
s^{2}=1 \text { or } 0(\bmod 4)}} h\left(q^{2}\left(s^{2}-4 n\right) / f^{2}\right) \\
& t_{h}=-(q-1) \sum_{\substack{d \mid n \\
0<d<\sqrt{n}}} d^{k-1} c_{q}\left(\Phi_{d+n / d}\right)
\end{aligned}
$$




$$
t_{p}=-\delta(n) \begin{cases}\frac{1}{2}(q-1)(q-2) n^{(\kappa-1) / 2}, & \nu=2 \\ \frac{1}{2}(q-1)^{2} q^{\nu / 2-1} n^{(\kappa-1) / 2}, & \nu \geq 4 \text { and even } \\ 0 \quad, & \nu \text { odd } .\end{cases}
$$

Here for $s \in Z, \Phi_{s}(X)=X^{2}-s X+n$, and

$$
c_{q}\left(\Phi_{s}\right)= \begin{cases}\sum_{\substack{x \bmod q^{\nu-1}-1 \\ \Phi_{s}(x) \equiv 0\left(\bmod q^{\nu}-2\right)}} \chi\left(\Phi_{s}(x) / q^{\nu-2}\right), & \nu \geq 3 \\ \sum_{\substack{x \bmod q \\ x \neq s(\bmod q)}} \chi\left(\Phi_{s}(x)\right), & \nu=2 .\end{cases}
$$

For $\Phi_{s}(X), \zeta$ and $\eta$ denote the two roots of $\Phi_{s}(X)=0$. s runs through all integers such that $s^{2}-4 n<0$, and $f$ runs through all positive integers such that $f^{2} \mid\left(s^{2}-4 n\right),(f, q)=1$, and $\left(s^{2}-4 n\right) / f^{2} \equiv 1$ or $0(\bmod 4)$. $h(4)$ denotes the class number of the order of $\boldsymbol{Q}(\sqrt{\Delta})$ with the discriminant $\Delta$. $d$ runs through all positive integer such that $d \mid n$ and $0<d<\sqrt{n} . \quad \delta(n)=1$ or 0 according as $n$ is a square or not.

Proof. We know by (2.2) that for $\alpha \in \prod_{p \neq q} \Xi_{p}(n) \times \Xi_{q}\left(\delta_{z} W \delta_{z} W\right) \times U_{\infty}$, $\chi_{1}(\alpha)$ depends only on the $U_{q}$-conjugacy class of the $q$-component $\alpha_{q}$ of $\alpha$ and $\chi_{1}(\alpha)=\chi_{1}\left(\alpha_{q}\right)$. For $\alpha \in \Xi\left(\delta_{x} W \delta_{x} W T_{n}\right)$, let $f_{\alpha}(X)$ denote the characteristic polynomial of $\alpha$. Then we see $f_{\alpha}(X)=q^{2 \nu+4} \Phi_{s}\left(q^{-\nu-2} X\right)$ for some $s \in Z$. First we treat the contribution $t_{e}$ from the elliptic elements. For $\alpha \in \Xi\left(\delta_{\chi} W \delta_{\chi} W T_{n}\right)$, let $\Phi_{s}(X)$ be as above, then $\alpha$ is an elliptic element if and only if $s^{2}-4 n<0$. For $\Phi_{s}(X)=X^{2}-s X+n \in Z[X]$, put $\Psi_{s}(X)$ $=q^{2 \nu+4} \Phi_{s}\left(q^{-\nu-2} X\right)$, then we have

$$
\begin{aligned}
\left\{\alpha \in \Xi\left(\delta_{\chi} W \delta_{\chi} W T_{n}\right) \mid f_{\alpha}\right. & \left.=\Psi_{s}\right\} \\
& =\bigcup_{\Lambda}\left\{\alpha \in \Xi\left(\delta_{\chi} W \delta_{\chi} W T_{n}\right) \mid f_{\alpha}=\Psi_{s}, \boldsymbol{Q}[\alpha] \cap R\left(q^{\nu}\right)=\varphi(\Lambda)\right\},
\end{aligned}
$$

where $\varphi$ is an isomorphism of $K\left(\Phi_{s}\right)$ to $\boldsymbol{Q}[\alpha]$ such that $\varphi(\tilde{X})=\alpha$, and $\Lambda$ runs through all $Z$-order of $K\left(\Psi_{s}\right)$ containing $\tilde{X}$. We note $\mid C_{p}(\alpha, \Lambda)$ $\cap \Xi_{p}(n) / \tilde{U}_{p} \mid=1$ for $p \neq q$ by Cor. 2.5, $\left|C_{\infty}(\alpha, \Lambda) \cap U_{\infty} / \tilde{U}_{\infty}\right|=2$ and

$$
\frac{\zeta^{\prime x-1}-\eta^{\alpha-1}}{\zeta^{\prime}-\eta^{\prime}} q^{-(2 \nu+4)(x / 2-1)}=\frac{\zeta^{x-1}-\eta^{\alpha-1}}{\zeta-\eta}
$$

for the two roots $\zeta^{\prime}$ and $\eta^{\prime}$ (resp. $\zeta$ and $\eta$ ) of $\Psi_{s}(X)=0$ (resp. $\Phi_{s}(X)=0$ ). By (2.4), Th. 2.4 and Prop. 2.6, we obtain 


$$
\begin{gathered}
t_{e}=-\frac{1}{2} \sum_{s} \frac{\zeta^{x-1}-\eta^{k-1}}{\zeta-\eta} \sum_{\Lambda} \frac{h\left(K\left(\Psi_{s}\right), \Lambda\right)}{\left[\Lambda^{\times}:\{ \pm 1\}\right]} c_{q}\left(s, \Lambda_{q}\right) \\
c_{q}\left(s, \Lambda_{q}\right)=\sum_{x} \chi_{1}\left(\varphi_{x}(\tilde{X})\right)+\sum_{y} \chi_{1}\left(\varphi_{y}^{\prime}(\tilde{X})\right),
\end{gathered}
$$

where $s$ runs through all integers such that $s^{2}-4 n<0$, and $\zeta, \eta$ denote the two roots of $\Phi_{s}(X)=0$. $\Lambda$ runs through all $Z$-order of $K\left(\Psi_{s}\right)$ containing $\tilde{X}$. Let $\Omega_{q}\left(\nu, \Psi_{s}, \Lambda\right)$ and $\Omega_{q}^{\prime}\left(\nu, \Psi_{s}, \Lambda\right)$ be as in Th. 2.4 and for $x \in \Omega_{q}\left(\nu, \Psi_{s}, \Lambda\right)\left(\operatorname{resp} . y \in \Omega_{q}^{\prime}\left(\nu, \Psi_{s}, \Lambda\right)\right)$ let $\varphi_{x}(\tilde{X})\left(\operatorname{resp} . \varphi_{y}(\tilde{X})\right)$ be as in Th. 2.4. Then $x$ (resp. $y$ ) runs through a complete system of representatives of $\left\{x \in \Omega_{q}\left(\nu, \Psi_{s}, \Lambda\right) \mid \varphi_{x}(\tilde{X}) \in \Xi_{q}\left(\delta_{x} W \delta_{x} W\right)\right\} \quad$ (resp. $\left\{y \in \Omega_{q}^{\prime}\left(\nu, \Psi_{s}, \Lambda\right) \mid \varphi_{y}^{\prime}(\tilde{X})\right.$ $\left.\in \Xi_{q}\left(\delta_{\chi} W \delta_{\chi} W\right)\right\}$ modulo $q^{\nu+\rho}$, where $\rho$ is a non-negative integer such that $\left[\Lambda_{q}: Z_{q}[\tilde{X}]\right]=q^{\rho}$. Now by the definition of $\Xi_{q}\left(\delta_{\chi} W \delta_{x} W\right)$, we see $\left[\Lambda_{q}: Z_{q}[\tilde{X}]\right]$ must be $q^{\nu+1}$, hence $\rho=\nu+1$. Assume $\Lambda_{q}$ is such an order. If $\left(\begin{array}{ll}a & b \\ c & d\end{array}\right)$ $\in \Xi_{q}\left(\delta_{\chi} W \delta_{x} W\right), v_{q}(b c)=3 \nu+2$, hence $\varphi_{y}^{\prime}(\tilde{X}) \notin \Xi_{q}\left(\delta_{x} W \delta_{z} W\right)$ for $y \in \Omega_{q}^{\prime}\left(\nu, \Psi_{s}, \Lambda\right)$. We note

$$
\Omega_{q}\left(\nu, \Psi_{s}, \Lambda\right)=\left\{q^{\nu+2} x \mid x \in Z_{q}, \Phi_{s}(x) \equiv 0\left(\bmod q^{\nu-2}\right)\right\}
$$

and for $x \in\left\{x \in Z_{q} \mid \Phi_{s}(x) \equiv 0\left(\bmod q^{\nu-2}\right)\right\}$, in the case where $\nu \geq 3(\operatorname{resp} . \nu=2)$, $\varphi_{x^{\prime}}(\tilde{X}), x^{\prime}=q^{\nu+2} x$, is contained in $\Xi_{q}\left(\delta_{x} W \delta_{x} W\right)$ if and only if $\Psi_{s}\left(x^{\prime}\right) \equiv 0$ $\left(\bmod q^{3 \nu+3}\right)\left(\operatorname{resp} . \Psi_{s}\left(x^{\prime}\right) \equiv 0\left(\bmod q^{9}\right)\right.$ and $\left.x \neq s(\bmod q)\right)$. The condition $\Psi_{s}\left(x^{\prime}\right) \equiv 0\left(\bmod q^{3 \nu+3}\right)$ is equivalent to $\Phi_{s}(x) \equiv 0\left(\bmod q^{\nu-1}\right)$. Since $\chi_{1}\left(\varphi_{x^{\prime}}(\tilde{X})\right)$. $=\chi\left(\Psi_{s}\left(q^{\nu+2} x\right) / q^{3 \nu+2}\right)=\chi\left(\Phi_{s}(x) / q^{\nu-2}\right)$, we obtain

$$
c_{q}\left(s, \Lambda_{q}\right)= \begin{cases}\sum_{\substack{x \bmod q^{\nu-1} \\ \Phi_{s}(x) \equiv 0\left(\bmod q^{\nu}-2\right)}} \chi\left(\Phi_{s}(x) / q^{\nu-2}\right), & \nu \geq 3 \\ \sum_{\substack{x \bmod q \\ x \neq s(\bmod q)}} \chi\left(\Phi_{s}(x)\right), & \nu=2 .\end{cases}
$$

We note for $K\left(\Psi_{s}\right)=\boldsymbol{Q}[X] /\left(\Psi_{s}(X)\right)$ and $K\left(\Phi_{s}\right)=\boldsymbol{Q}[Y] /\left(\Phi_{s}(Y)\right)$ there exists. an isomorphism $\lambda$ of $K\left(\Psi_{s}\right)$ to $K\left(\Phi_{s}\right)$ such that $\lambda(\tilde{X})=q^{\nu+2} \tilde{Y}$, and for a $Z$ order $\Lambda$ of $K\left(\Psi_{s}\right),\left[\Lambda_{q}: Z_{q}[\tilde{X}]\right]=q^{\nu+1}$ if and only if $\left[Z_{q}[\tilde{Y}]: \lambda\left(\Lambda_{q}\right)\right]=q$. Our assertion for $t_{e}$ follows from this. The contribution from the hyperbolic elements can be treated in the same way by noting that $\left|C_{\infty}(\alpha, \Lambda) \cap U_{\infty} / \tilde{U}_{\infty}\right|$ $=1$ for a hyperbolic element $\alpha$ fixing a cusp of $\Gamma_{0}\left(q^{\nu}\right)$ and that the class number of the order $\Lambda$ of $\boldsymbol{Q} \oplus \boldsymbol{Q}$ such that $[\boldsymbol{Z} \oplus \boldsymbol{Z}: \Lambda]=m$ is given by $\varphi(m)$ with the Euler function $\varphi$, and we obtain 


$$
t_{h}=-\sum_{\substack{d \mid n \\ 0<d<\sqrt{n}}} \frac{d^{x-1}}{n / d-d} c_{q}\left(\Phi_{d+n / d}\right) \sum_{\substack{f \mid(n / d-d) \\(f, q)=1}} \varphi(q(n / d-d) / f) .
$$

Thus we have

$$
t_{h}=-(q-1) \sum_{\substack{d i n \\ 0<d<\sqrt{n}}} d^{x-1} c_{q}\left(\Phi_{d+n / d}\right)
$$

Now we shall calculate the contribution $t_{p}$ from the parabolic elements. If $\Xi\left(\delta_{\chi} W \delta_{\chi} W T_{n}\right)_{p}$ is not empty, $n$ is a square, and the characteristic polynomial $f_{\alpha}$ of an element $\alpha$ of $\Xi\left(\delta_{\chi} W \delta_{\alpha} W T_{n}\right)_{p}$ is either $\left(X-q^{\nu+2} \sqrt{n}\right)^{2}$ or $\left(X+q^{\nu+2} \sqrt{n}\right)^{2}$. Since the contributions from the parabolic elements with the characteristic polynomial $\left(X-q^{\nu+2} \sqrt{n}\right)^{2}$ and from those with the characteristic polynomial $\left(X+q^{\nu+2} \sqrt{n}\right)^{2}$ are the same, it is enough to consider only the parabolic element $\alpha$ with $f_{\alpha}(X)=\left(X-q^{\nu+2} \sqrt{n}\right)^{2}$. Put $\Psi(X)$ $=\left(X-q^{\nu+2} \sqrt{n}\right)^{2}$ and $\Phi(X)=(X-\sqrt{n})^{2}$, then we have

$$
\begin{aligned}
& \left\{\alpha \mid \alpha \in \Xi\left(\delta_{\chi} W \delta_{\chi} W T_{n}\right)_{p}, f_{\alpha}=\Psi\right\} \\
& \quad=\bigcup_{\Lambda}\left\{\alpha \mid \alpha \in \Xi\left(\delta_{\chi} W \delta_{\chi} W T_{n}\right)_{p}, f_{\alpha}=\Psi, Q[\alpha] \cap R\left(q^{\nu}\right)=\varphi(\Lambda)\right\},
\end{aligned}
$$

where $\varphi$ is the isomorphism of $K(\Psi)$ to $\boldsymbol{Q}[\alpha]$ such that $\varphi(\tilde{X})=\alpha$ and $\Lambda$ runs through all $Z$-order of $K(\Psi)$ such that $\Lambda \ni \tilde{X}$. As in the case of elliptic elements, we see $\left[\Lambda_{q}: Z_{q}[\tilde{X}]\right]$ must be $q^{\nu+1}$, and for such an order $\varphi_{y}^{\prime}(X) \notin \Xi_{q}\left(\delta_{x} W \delta_{x} W\right)$ for $y \in \Omega_{q}^{\prime}(\nu, \Psi, \Lambda)$. If $\nu$ is odd, for $x \in Z_{q}, \Psi(x) \equiv 0$ $\left(\bmod q^{3 \nu+2}\right)$ implies $\Psi(x) \equiv 0\left(\bmod q^{3 \nu+3}\right)$, hence $\Xi\left(\delta_{\chi} W \delta_{\chi} W T_{n}\right)_{p}$ is empty in this case. Assume $\nu$ is even. Put $\Omega=\left\{x \in Z_{q} \mid \Psi(x) \equiv 0\left(\bmod q^{3 \nu+2}\right), \Psi(x) \not \equiv 0\right.$ $\left.\left(\bmod q^{3 \nu+3}\right)\right\}$ for $\nu \geq 4$ and $\Omega=\left\{x \in Z_{q} \mid \Psi(x) \equiv 0\left(\bmod q^{8}\right), \Psi(x) \not \equiv 0\left(\bmod q^{9}\right)\right.$, $\left.x \neq \equiv 2 q^{4} \sqrt{n}\left(\bmod q^{5}\right)\right\}$ for $\nu=2$. Then $\left\{\varphi_{x}(\tilde{X}) \mid x \in \Omega\left(\bmod q^{2 \nu+1}\right)\right\}$ gives a complete system of representatives of $C_{q}(\alpha, \Lambda) \cap \Xi_{q}\left(\delta_{z} W \delta_{z} W\right) / \tilde{U}_{q}$ for $\alpha$ with the minimal polynomial $\Psi$. We see $\chi_{1}\left(\varphi_{x}(\tilde{X})\right)=1$ for $x \in \Omega$ and $\left|\Omega\left(\bmod q^{2 \nu+1}\right)\right|$ $=q^{\nu / 2}(1-1 / q)$ if $\nu \geq 4$ and $\left|\Omega\left(\bmod q^{5}\right)\right|=(q-2)$ if $\nu=2$. Let $\alpha$ $\in \Xi\left(\delta_{x} W \delta_{x} W T_{n}\right)_{p}$ be an element such that $f_{\alpha}=\Psi, Q[\alpha] \cap R\left(q^{\nu}\right)=\varphi(\Lambda)$, and put $[\Lambda: Z[\tilde{X}]]=m q^{\nu+1}$ with a positive integer $m$ prime to $q$, then $m(\alpha)$ $=q^{\nu+1} m / q^{\nu+2} \sqrt{n}=m /(q \sqrt{n})$. We note $\left|C_{\infty}(\alpha, \Lambda) \cap U_{\infty} / \tilde{U}_{\infty}\right|=2$ for $\alpha$ with the minimal polynomial $\Psi$ and $h(K(\Psi), \Lambda)=1$ for any $Z$-order $\Lambda$ of $K(\Psi)$. Thus we obtain

$$
t_{p}=-\lim _{s \rightarrow 0} \frac{s}{2} \sum_{\substack{m=1 \\(m, q)=1}}^{\infty}\left(\frac{q \sqrt{n}}{m}\right)^{1+s}\left|\Omega\left(\bmod q^{2 \nu+1}\right)\right| n^{\kappa / 2-1}
$$




$$
= \begin{cases}-\frac{1}{2}(q-1)(q-2) n^{(\kappa-1) / 2}, & \nu=2 \\ -\frac{1}{2}(q-1)^{2} q^{\nu / 2-1} n^{(\kappa-1) / 2}, & \nu \geq 4 \text { and even } .\end{cases}
$$

Thus the proof of our theorem is completed.

Corollary 2.8. If $\nu$ is odd, $\nu \geq 3$, and $q \geq 5$, then one has

$$
\operatorname{dim} S_{\mathrm{I}}+\operatorname{dim} S_{\mathrm{III}}=\operatorname{dim} S_{\mathrm{II}}+\operatorname{dim} S_{\mathrm{II}_{\chi}}
$$

Proof. Since we have

$$
\operatorname{tr} \delta_{\chi} W \delta_{\chi} W \mid S_{\kappa}\left(\Gamma_{0}\left(q^{\nu}\right)\right)=\operatorname{dim} S_{\mathrm{I}}+\operatorname{dim} S_{\mathrm{III}}-\operatorname{dim} S_{\mathrm{II}}-\operatorname{dim} S_{\mathrm{II}_{\chi}},
$$

and $\Xi\left(\delta_{x} W \delta_{x} W\right)_{h}$ is empty, it is enough to prove $t_{e}=0$. The characteristic polynomial of an element in $\Xi\left(\delta_{x} W \delta_{x} W\right)$ is either $X^{2}+q^{2 \nu+4}$ or $X^{2} \pm q^{\nu+2} X+q^{2 \nu+4}$. Let $t_{1}$ (resp. $t_{2}$ ) denote the contribution from the elliptic elements with the characteristic polynomial $X^{2}+q^{2 \nu+4}$ (resp. $X^{2}+q^{\nu+2} X$ $+q^{2 \nu+4}$ or $\left.X^{2}-q^{\nu+2} X+q^{2 \nu+4}\right)$. Put $\Phi(X)=X^{2}+1$. If the equation $\Phi(X) \equiv 0$ $\left(\bmod q^{\nu-2}\right)$ has a solution, then $\Phi(X)=(X-\alpha)(X-\beta)$ with some $\alpha, \beta \in Z_{q}$. Since we have $\alpha-\beta \neq 0(\bmod q)$, all the solutions of $\Phi(X) \equiv 0\left(\bmod q^{\nu-2}\right)$ are given by $\left\{\alpha+q^{\nu-2} \alpha^{\prime} \mid \alpha \in Z_{q}\right\} \cup\left\{\beta+q^{\nu-2} \beta^{\prime} \mid \beta^{\prime} \in Z_{q}\right\}$. Hence we obtain

$$
\begin{aligned}
c_{q}(\Phi) & =\sum_{\alpha^{\prime} \bmod q} \chi\left(\alpha^{\prime}(\beta-\alpha)\right)+\sum_{\beta^{\prime} \bmod q} \chi\left((\alpha-\beta) \beta^{\prime}\right) \\
& =0 .
\end{aligned}
$$

Therefore $t_{1}=0$ and we can prove $t_{2}=0$ in the same way.

§3. A formula for $\operatorname{tr} \delta_{x} W \delta_{x} T_{n}$

We use the same notation as in $\S 2$. For $\nu \geq 2$, let $\Xi_{q}\left(\delta_{x} W \delta_{x}\right)$ denote the subset of $R_{q}(\nu)$ given by

$$
\Xi_{q}\left(\delta_{\chi} W \delta_{x}\right)= \begin{cases}\left(\begin{array}{cc}
q^{\nu+1} Z_{q}^{\times} & q^{2} Z_{q}^{\times} \\
q^{\nu+2} Z_{q}^{\times} & q^{\nu+1} Z_{q}^{\times}
\end{array}\right), & \nu \geq 3 \\
\left\{g \in\left(\begin{array}{ll}
q^{3} Z_{q}^{\times} & q^{2} Z_{q} \\
q^{4} Z_{q}^{\times} & q^{3} Z_{q}^{\times}
\end{array}\right) v_{q}(\operatorname{det} g)=6\right\}, & \nu=2,\end{cases}
$$

then $\Xi_{q}\left(\delta_{x} W \delta_{\chi}\right)$ is a union of $U_{q}$-double cosets. For $i, j, 1 \leq i, j \leq q-1$, put

$$
\beta_{i j}=\left(\begin{array}{ll}
q & i \\
0 & q
\end{array}\right)\left(\begin{array}{rr}
0 & -1 \\
q^{\nu} & 0
\end{array}\right)\left(\begin{array}{ll}
q & j \\
0 & q
\end{array}\right)
$$




$$
=\left(\begin{array}{cc}
i q^{\nu+1} & i j q^{\nu}-q^{2} \\
q^{\nu+2} & j q^{\nu+1}
\end{array}\right)
$$

Multiplying by $\left(\begin{array}{lr}0 & -1 \\ q^{\nu} & 0\end{array}\right)^{-1}$ on both sides of (2.1), we obtain

LEMMA 3.1. Assume $\nu \geq 2$, and put $\beta_{i j}=\left(\begin{array}{cc}i q^{\nu+1} & i j q^{\nu}-q^{2} \\ q^{\nu+2} & j q^{\nu+1}\end{array}\right), 1 \leq i, j$ $\leq q-1$, then one has

$$
\Xi_{q}\left(\delta_{z} W \delta_{z}\right)=\bigcup_{i, j=1}^{q-1} U_{q} \beta_{i j}
$$

and the right hand side is a disjoint union.

By virtue of this lemma, the following lemma can be proved in the same way as Lemma 2.3.

LEMMA 3.2. Let $n$ be a positive integer prime to $q$, and assume $\nu \geq 2$. Let $\left(\prod_{p \neq q} \Xi_{p}(n) \times U_{q} \times U_{\infty}\right) \cap G L_{2}(\boldsymbol{Q})=\bigcup_{\lambda=1}^{d} \Gamma_{0}\left(q^{\nu}\right) \beta_{\lambda}$ be a disjoint union, then one has

$$
\begin{gathered}
\left(\prod_{p \neq q} \Xi_{p}(n) \times \Xi_{q}\left(\delta_{\chi} W \delta_{\chi}\right) \times U_{\infty}\right) \cap G L_{2}(\mathbf{Q}) \\
=\bigcup_{i, j=1}^{q-1} \bigcup_{\lambda=1}^{d} \Gamma_{0}\left(q^{\nu}\right) \beta_{i j} \beta_{\lambda},
\end{gathered}
$$

and the right hand side is a disjoint union.

For $\alpha_{q}=\left(\begin{array}{ll}a & b \\ c & d\end{array}\right) \in \Xi_{q}\left(\delta_{x} W \delta_{x}\right)$, we put

$$
\chi_{2}\left(\alpha_{q}\right)=\chi\left(a d / q^{2 \nu+2}\right) .
$$

Then for $\gamma_{q} \in U_{q}$, we have

$$
\chi_{2}\left(\gamma_{q}^{-1} \alpha_{q} \gamma_{q}\right)=\chi_{2}\left(\alpha_{q}\right) .
$$

For $\alpha \in \prod_{p \neq q} \Xi_{p}(n) \times \Xi_{q}\left(\delta_{x} W \delta_{x}\right) \times U_{\infty}$, we define

$$
\chi_{2}(\alpha)=\chi_{2}\left(\alpha_{q}\right)
$$

where $\alpha_{q}$ denotes the $q$-component of $\alpha$. Then we can verify

$$
\chi_{2}\left(\gamma \alpha \gamma^{\prime}\right)=\chi_{2}(\alpha) \quad \text { for } \gamma, \gamma^{\prime} \in \Gamma_{0}\left(q^{\nu}\right) .
$$

For a positive integer $n$ prime to $q$, put 


$$
\Xi\left(\delta_{x} W \delta_{z} T_{n}\right)=\left(\prod_{p \neq q} \Xi_{p}(n) \times \Xi_{q}\left(\delta_{z} W \delta_{z}\right) \times U_{\infty}\right) \cap G L_{2}(Q)
$$

For $f \in S_{x}\left(\Gamma_{0}\left(q^{\nu}\right)\right)$, we define

$$
\left.f\right|_{\chi} \Xi\left(\delta_{\chi} W \delta_{\chi} T_{n}\right)=\frac{\chi(n)}{W(\chi)^{2}} n^{\alpha / 2-1} \sum_{\alpha \in \Gamma_{0}\left(q^{\nu}\right) \backslash \delta\left(\delta_{\chi} W \delta_{\chi} T_{n}\right)} \chi_{2}(\alpha) f \mid[\alpha]_{\kappa},
$$

then by (3.2) and Lemma 3.2, we have

$$
f\left|\delta_{\chi} W \delta_{\chi} T_{n}=f\right|_{\chi} \Xi\left(\delta_{x} W \delta_{\chi} T_{n}\right)
$$

By Eichler-Selberg's trace formula, we obtain

$$
\begin{gathered}
\operatorname{tr} \delta_{x} W \delta_{x} T_{n} \mid S_{k}\left(\Gamma_{0}\left(q^{\nu}\right)\right)=\frac{\chi(n)}{W(\chi)^{2}}\left(t_{e}+t_{h}+t_{p}\right) \\
t_{e}=-\frac{1}{4} \sum_{\alpha \in B\left(\delta_{x} W \delta_{x} T_{n}\right)_{e} / \Gamma_{0\left(q^{\nu}\right)}} \chi_{2}(\alpha) \frac{1}{[\Gamma(\alpha):\{ \pm 1\}]} \frac{\zeta_{\alpha}^{\kappa-1}-\eta_{\alpha}^{\alpha-1}}{\zeta_{\alpha}-\eta_{\alpha}} q^{-(\nu+4)(\alpha / 2-1)} \\
t_{h}=-\frac{1}{2} \sum_{\alpha \in B\left(\delta_{x} W \delta_{x} T_{n}\right)_{n} / \Gamma_{0}\left(q^{\nu}\right)} \chi_{2}(\alpha) \frac{\left(\min \left(\left|\zeta_{\alpha}\right|,\left|\eta_{\alpha}\right|\right)\right)^{\alpha-1}}{\left|\zeta_{\alpha}-\eta_{\alpha}\right|} q^{-(\nu+4)(\alpha / 2-1)} \\
t_{p}=-\lim _{s \rightarrow 0} \frac{s}{8} \sum_{\alpha \in B\left(\delta_{x} W \delta_{x} T_{n}\right)_{p} / \widetilde{\Gamma}_{0}\left(q^{\nu}\right)} \chi_{2}(\alpha) \frac{1}{|m(\alpha)|^{1+s}} n^{\alpha / 2-1},
\end{gathered}
$$

where $\Xi\left(\delta_{\chi} W \delta_{\chi} T_{n}\right)_{e}$ (resp. $\Xi\left(\delta_{\chi} W \delta_{x} T_{n}\right)_{h}$; resp. $\left.\Xi\left(\delta_{\chi} W \delta_{x} T_{n}\right)_{p}\right)$ denotes the set of all elliptic elements (resp. hyperbolic elements fixing cusps of $\Gamma_{0}\left(q^{\nu}\right)$; resp. parabolic elements fixing cusps of $\left.\Gamma_{0}\left(q^{\nu}\right)\right)$ in $\Xi\left(\delta_{x} W \delta_{x} T_{n}\right)$ and the other notation is the same as in (2.4). We can express $\operatorname{tr} \delta_{\chi} W \delta_{\chi} T_{n} \mid S_{\kappa}\left(\Gamma_{0}\left(q^{\nu}\right)\right)$ in a more explicit form, namely, we can prove

THEOREM 3.3. Let $n$ be a positive integer prime to $q$, and $\kappa$ be an even positive integer. Assume $\nu \geq 2$, then one has

$$
\operatorname{tr} \delta_{\chi} W \delta_{\chi} T_{n} \mid S_{\kappa}\left(\Gamma_{0}\left(q^{\nu}\right)\right)=\frac{\chi(n)}{W(\chi)^{2}}\left(t_{e}+t_{h}+t_{p}\right)
$$

where

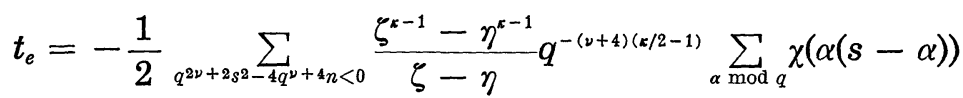

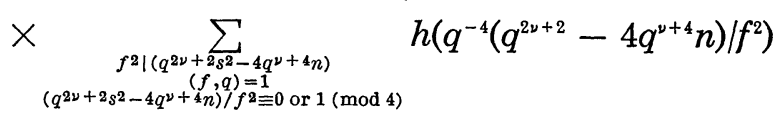




$$
\begin{aligned}
& t_{h}= \begin{cases}-(q-1) q^{\nu / 2-1} \sum_{\substack{d \mid n \\
0<d<\sqrt{n} \\
d+n / d \leq 0\left(\bmod q^{\nu / 2}-1\right)}} d^{k-1} \sum_{\alpha \bmod q} \chi\left(\alpha\left((d+n / d) / q^{\nu / 2-1}-\alpha\right)\right) \\
0 & , \text { if } \nu \text { is even }\end{cases} \\
& t_{p}= \begin{cases}0, & , \text { if } \nu \text { is odd } \\
-\frac{1}{2} \delta(n)(q-1) n^{(k-1) / 2} \sum_{\alpha \bmod q} \chi(\alpha(2 \sqrt{n}-\alpha)), & \nu=2 .\end{cases}
\end{aligned}
$$

Here $s$ runs through all integers such that $q^{2 \nu+2} s^{2}-4 q^{\nu+4} n<0$, and $\zeta, \eta$ are the solutions of $X^{2}-q^{\nu+1} s X+q^{\nu+4} n=0$. $f$ runs through all positive integers prime to $q$ such that $f^{2} \mid\left(q^{2 \nu+2} s^{2}-4 q^{\nu+4} n\right)$ and $\left(q^{2 \nu+2} s^{2}-4 q^{\nu+4} n\right) \mid f^{2} \equiv 0$ or $1(\bmod 4) . d$ runs through all positive integers such that $d \mid n, 0<d<\sqrt{n}$, and $d+n / d \equiv 0\left(\bmod q^{\nu / 2-1}\right)$.

Proof. As in the case of $\chi_{1}$, for $\alpha \in \prod_{p \neq q} \Xi_{p}(n) \times \Xi_{q}\left(\delta_{x} W \delta_{x}\right) \times U_{\infty}$, $\chi_{2}(\alpha)$ depends only on the $U_{q}$-conjugacy class of the $q$-component $\alpha_{q}$ of $\alpha$ and $\chi_{2}(\alpha)=\chi_{2}\left(\alpha_{q}\right)$. By the definition of $\Xi\left(\delta_{\chi} W \delta_{x} T_{n}\right)$, the characteristic polynomial $f_{\alpha}$ of $\alpha$ in $\Xi\left(\delta_{x} W \delta_{x} T_{n}\right)$ is of the form $\Psi_{s}(X)=X^{2}-q^{\nu+1} s X+q^{\nu+4} n$ for some integer $s$. Let $\Lambda$ be a $Z$-order of $K\left(\Psi_{s}\right)$ such that $\boldsymbol{Q}[\alpha] \cap R\left(q^{\nu}\right)$ $=\varphi(\Lambda)$, then we see $\left[\Lambda_{q}: Z_{q}[\tilde{X}]\right]$ must be $q^{2}$, where $\varphi$ is an isomorphism of $K\left(\Psi_{s}\right)$ to $\boldsymbol{Q}[\alpha]$ such that $\varphi(\tilde{X})=\alpha$. First we calculate the contribution $t_{e}$ from the elliptic elements. An elements $\alpha \in \Xi\left(\delta_{\chi} W \delta_{x} T_{n}\right)$ with $f_{\alpha}=\Psi_{s}$ is elliptic if and only if $q^{2 \nu+2} s^{2}-4 q^{\nu+4} n<0$. Let $\Lambda$ be a $Z$-order of $K\left(\Psi_{s}\right)$ such that $\left[\Lambda_{q}: Z_{q}[\tilde{X}]\right]=q^{2}$. For $x \in \Omega_{q}\left(\nu, \Psi_{s}, \Lambda\right)\left(\right.$ resp. $y \in \Omega_{q}^{\prime}\left(\nu, \Psi_{s}, \Lambda\right)$ ), we see $v_{q}(x)=v_{q}\left(q^{\nu+1} s-x\right)=\nu+1 \quad\left(\right.$ resp. $\left.v_{q}(y)=v_{q}\left(q^{\nu+1} s-y\right)=\nu+1\right)$ if $\varphi_{x}(\tilde{X}) \in \Xi_{q}\left(\delta_{x} W \delta_{x}\right)$ (resp. $\varphi_{y}^{\prime}(\tilde{X}) \in \Xi_{q}\left(\delta_{x} W \delta_{x}\right)$ ). If $\nu \geq 3, \varphi_{y}^{\prime}(\tilde{X}) \notin \Xi_{q}\left(\delta_{x} W \delta_{x}\right)$ for $y \in \Omega_{q}^{\prime}\left(\nu, \Psi_{s}, \Lambda\right)$, and put $\Omega=\left\{q^{\nu+1} x^{\prime} \mid x^{\prime} \in Z_{q}, x^{\prime} \not \equiv s(\bmod q)\right\}$, then $\left\{\varphi_{x}(\tilde{X}) \mid x\right.$ $\left.\in \Omega\left(\bmod q^{\nu+2}\right)\right\}$ gives a complete system of representatives of $\left\{\alpha \in \Xi_{q}\left(\delta_{x} W \delta_{x}\right) \mid f_{\alpha}\right.$ $\left.=\Psi_{s}\right\} / \tilde{U}_{q}$ and $\chi_{2}\left(\varphi_{x}(\tilde{X})\right)=\chi\left(x^{\prime}\left(s-x^{\prime}\right)\right)$. If $\nu=2$, put $\Omega=\left\{q^{3} x^{\prime} \mid x^{\prime} \in Z_{q}^{\times}, x^{\prime} \not \equiv s\right.$ $\left.(\bmod q), x^{\prime 2}-s x^{\prime}+n \neq \equiv 0(\bmod q)\right\}$ and $\Omega^{\prime}=\left\{q^{3} y^{\prime} \mid y^{\prime} \in Z_{q}, y^{\prime 2}-s y^{\prime}+n \equiv 0\right.$ $(\bmod q)\}$, then we see $\left\{\varphi_{x}(\tilde{X}) \mid x \in \Omega\left(\bmod q^{4}\right)\right\} \cup\left\{\varphi_{y}^{\prime}(\tilde{X}) \mid y \in \Omega^{\prime}\left(\bmod q^{4}\right)\right\}$ gives a complete system of representatives of $\left\{\alpha \in \Xi_{q}\left(\delta_{x} W \delta_{x}\right) \mid f_{\alpha}=\Psi_{s}\right\} / \tilde{U}_{q}$ and $\chi_{2}\left(\varphi_{x}(\tilde{X})\right)=\chi\left(x^{\prime}\left(s-x^{\prime}\right)\right), \chi_{2}\left(\varphi_{y}(\tilde{X})\right)=\chi\left(y^{\prime}\left(s-y^{\prime}\right)\right)$. Our assertion on $t_{e}$ follows from this in the same way as in the case of $\delta_{z} W \delta_{\chi} W T_{n}$. If $\nu$ is odd, we see easily $\Xi\left(\delta_{x} W \delta_{x} T_{n}\right)_{h}$ is empty, hence $t_{h}=0$. Assume $\nu$ 'is even, and let $\alpha$ be an element of $\Xi\left(\delta_{\alpha} W \delta_{\chi} T_{n}\right)_{h}$ with $f_{\alpha}=\Psi_{s}$ for some integer $s$. Let $q^{\nu_{1}} n_{1}$ and $q^{\nu_{2}} n_{2}$ be the solutions of $\Psi_{s}(X)=0$, then $\nu_{1}+\nu_{2}=\nu+4$ and $n=n_{1} n_{2}$. Since $\left(q^{\nu_{1}} n_{1}-q^{\nu_{2}} n_{2}\right)^{2}=\left(q^{\nu+1} s\right)^{2}-4 q^{\nu+4} n=q^{\nu+4}\left(q^{\nu-2} s^{2}-4 n\right), \nu_{1}=\nu_{2}$ 
$=(\nu+4) / 2$ and $s=q^{(\nu+4) / 2}\left(n_{1}+n_{2}\right)$. Hence $\Psi_{s}$ is of the form $X^{2} \pm q^{(\nu+4) / 2}$ $(d+n / d) X+q^{\nu+4} n$ for $d$, where $d$ satisfies $d \mid n, 0<d<\sqrt{n}$ and $d+n / d$ $\equiv 0\left(\bmod q^{\nu / 2-1}\right)$. Thus our assertion on $t_{h}$ can be proved in the same way as above. Finally we treat the contribution from the parabolic elements. If $\left(q^{\nu+2} s\right)^{2}-4 q^{\nu+4} n=0$ for an integer $s, \nu$ must be 2 since we assume $n$ is prime to $q$. Hence $\Xi\left(\delta_{\chi} W \delta_{\chi} T_{n}\right)_{p}$ is empty if $\nu \geq 3$, and $t_{p}=0$. Assume $\nu=2$ and $n$ is a square, then the characteristic polynomial $f_{\alpha}$ of $\alpha$ in $\Xi\left(\delta_{\chi} W \delta_{x} T_{n}\right)_{p}$ is of the form $\Psi_{s}(X)=X^{2}-q^{3} s X+q^{6} n$ for $s= \pm 2 \sqrt{n}$. Let $\Lambda$ be a $Z$-order of $K\left(\Psi_{s}\right)$ such that $\boldsymbol{Q}[\alpha] \cap R\left(q^{\nu}\right)=\varphi(\Lambda)$, then $\left[\Lambda_{q}: Z_{q}[\tilde{X}]\right]=q^{2}$. If we put $[\Lambda: Z[\tilde{X}]]=m q^{2}$ with $m$ prime to $q$, then $m(\alpha)=m /(\sqrt{n} q)$. In the same way as above, we obtain

$$
\begin{aligned}
t_{p} & =-\lim _{s \rightarrow 0} \frac{s}{2} n^{\kappa / 2-1} \sum_{\substack{m=1 \\
(m, q)=1}}^{\infty}\left(\frac{\sqrt{n} q}{m}\right)^{1+s} \sum_{\alpha \bmod q} \chi(\alpha(2 \sqrt{n}-\alpha)) \\
& =-\frac{1}{2}(q-1) n^{(\kappa-1) / 2} \sum_{\alpha \bmod q} \chi(\alpha(2 \sqrt{n}-\alpha)) .
\end{aligned}
$$

Thus the proof of our theorem is completed.

\section{§4. The case $q^{\nu}=11^{3}$}

In this section, we shall make some numerical observation on $S_{2}^{0}\left(\Gamma_{0}\left(11^{3}\right)\right)$, which is of dimension 100 , using the formulae obtained in $\S 2$ and $\S 3$. As was shown in $\S 1, S_{2}^{0}\left(\Gamma_{0}\left(11^{3}\right)\right)$ decomposes into 4 subspaces $S_{\mathrm{I}}$, $S_{\text {II }}, S_{\text {II }}$, and $S_{\text {III. }}$ We find $\operatorname{dim} S_{\text {I }}=15, \operatorname{dim} S_{\text {II }}=\operatorname{dim} S_{\text {II }_{x}}=25$, and $\operatorname{dim} S_{\text {III }}$ $=35$. Now we take the space $S_{\mathrm{I}}$ and give the characteristic polynomials of the Hecke operators $T_{n}$ acting on $S_{\mathrm{I}}$ for several $n$, whose roots give $n$-th Fourier coefficients $a_{n}$ for some primitive form $f(z)=\sum_{n=1}^{\infty} a_{n} e^{2 \pi i n z}$ in

\begin{tabular}{|c|c|c|c|}
\hline \multirow{2}{*}{$n$} & \multirow{2}{*}{$\chi(n)$} & \multicolumn{2}{|c|}{ characteristic polynomial of $T_{n}$} \\
\hline & & $S_{\mathrm{I}_{1}}$ & $S_{\mathrm{I}_{2}}$ \\
\hline 2 & -1 & $X^{5}$ & $\begin{array}{c}X^{10}-16 X^{8}+98 X^{6}-285 X^{4} \\
+390 X^{2}-199\end{array}$ \\
\hline 3 & +1 & $\begin{array}{l}X^{5}+5 X^{4}-X^{3}-34 X^{2} \\
\quad-39 X+1\end{array}$ & $\begin{array}{l}\left(X^{5}+X^{4}-4 X^{3}-3 X^{2}\right. \\
\quad+3 X+1)^{2}\end{array}$ \\
\hline 5 & +1 & $\begin{array}{l}X^{5}+4 X^{4}-9 X^{3}-27 X^{2} \\
+31 X+23\end{array}$ & $\begin{array}{l}\left(X^{5}+6 X^{4}+10 X^{3}+X^{2}\right. \\
\quad-6 X-1)^{2}\end{array}$ \\
\hline 199 & +1 & $\begin{array}{l}X^{5}+23 X^{4}-400 X^{3} \\
\quad-5811 X^{2}+52209 X \\
\quad+120077\end{array}$ & $\begin{array}{l}\left(X^{5}+56 X^{4}+502 X^{3}\right. \\
\quad-20441 X^{2}-426566 X \\
\quad-2148299)^{2}\end{array}$ \\
\hline
\end{tabular}
$S_{\text {I. }}$ 
For an explanation of this table, we remark the space $S_{\mathrm{I}}$ decomposes into the 5-dimensional space $S_{\mathrm{I}_{1}}$ and the 10-dimensional space $S_{\mathrm{I}_{2}}$. As was proved in [13], we have ten primitive forms in $S_{2}^{0}\left(\Gamma_{0}\left(11^{3}\right)\right)$, which correspond to the $L$-functions of $Q(\sqrt{-11})$ with Grössencharacters. By the following lemma, we know five such primitive forms spann the space $S_{\mathrm{I}_{1}}$ and the remaining five primitive forms are contained in $S_{\mathrm{III}}$.

LEMMA 4.1. Let $q$ be a prime such that $q \equiv 3(\bmod 4), q>3$, and $\kappa$ be an even positive integer. Let $\lambda$ be a Grössencharacter of $\boldsymbol{Q}(\sqrt{-q})$ with the conductor $(q)$ which satisfies

$$
\lambda((a))=\left(\frac{a}{|a|}\right)^{\kappa-1}
$$

for $a \in \boldsymbol{Q}(\sqrt{-q})$ with $a \equiv 1 \bmod ^{\times}(q)$ and $\chi(a) \lambda((a))=1$ for $a \in Z$. For an integer $a$ in $Q(\sqrt{-q})$, put $\lambda_{0}(a)=\lambda((a))\left(\frac{a}{|a|}\right)^{-(x-1)}$ and let $x_{0}$ be a rational integer such that

$$
\lambda_{0}(1+a \sqrt{-q})=e^{2 \pi i a x_{0} / q}
$$

for $a \in Z$. Then the primitive cusp form $f_{\lambda}(z)=\sum_{\mathfrak{a}} \lambda(\mathfrak{a}) N(\mathfrak{a})^{(\boldsymbol{k}-1) / 2} e^{2 \pi i N(\mathfrak{a}) z}$ corresponding to $\lambda$ is contained in $S_{k}^{0}\left(\Gamma_{0}\left(q^{3}\right)\right)$ and satisfies

$$
f_{\lambda} \mid W=(-i)^{x} \chi(-2) \chi\left(x_{0}\right) f_{\lambda}
$$

Proof. Comparing the functional equation of the $L$-function of $\lambda$ with that of cusp forms in $S_{*}^{0}\left(\Gamma_{0}\left(q^{3}\right)\right)$, we obtain

$$
f_{\lambda} \mid W=(-i)^{\star} T(\lambda) f_{\lambda},
$$

where $T(\lambda)=\frac{1}{q} \sum_{a \bmod (q)} \lambda_{0}(a) e^{2 \pi i \operatorname{tr}(a / q \sqrt{-q)}}$. By an easy calculation, we have $T(\lambda)=\chi(-2) \chi\left(x_{0}\right)$, and this completes the proof.

Hereafter we restrict our discussion to the 10-dimensional space $S_{\mathrm{I}_{2}}$, which is closely related to Shimura's theory on the construction of class fields over real quadratic fields. Let us fix a primitive form $f(z)$ $=\sum_{n=1}^{\infty} a_{n} e^{2 \pi i n z} \in S_{\mathrm{I}_{2}}$, and let $K$ denote the field generated over $\boldsymbol{Q}$ by $a_{n}$ for all $n$. Then as the table shows, $K$ and $f(z)$ satisfy the assumption (*) in $\S 1$. Let $F_{11}$ be the maximal real subfield of $Q\left(e^{2 \pi i / 11}\right)$, and put $\alpha_{0}$ $=e^{2 \pi i / 11}+e^{-2 \pi i / 11}$. Since $\sqrt{3-\alpha_{0}}$ satisfies $X^{10}-16 X^{8}+98 X^{6}-285 X^{4}$ $+390 X^{2}-199=0$, we may put $a_{2}=\sqrt{3-\alpha_{0}}$ and $K=F_{11}\left(\sqrt{3-\alpha_{0}}\right)$. Then 
we find

$$
\begin{aligned}
a_{2} & =\sqrt{3-\alpha_{0}}, \quad a_{3}=\alpha_{0}, \quad a_{5}=\alpha_{0}^{4}-4 \alpha_{0}^{2}+1, \\
a_{199} & =-12 \alpha_{0}^{4}-\alpha_{0}^{3}+44 \alpha_{0}^{2}-9 \alpha_{0}-33 .
\end{aligned}
$$

(These Fourier coefficients are obtained from $\operatorname{tr} T_{n}$ for several composite integers $n$, which are not given here.) We remark $f(z)$ and its companions $f_{o}(z)=\sum_{n=1}^{\infty} a_{n}^{a} e^{2 \pi i n z}$ spann the space $S_{\mathrm{I}_{2}}$, where $\sigma$ runs over all isomorphisms of $K$ into $C$, and the invariant subfield $F$ of $K$ under $\rho$ which is defined in $\S 1$ is $F=F_{11}$. In the following we follow the notation in $\S 1$. Since $f$ satisfies $(*)$, we can associate with $f$ a triple $(A, \mu, \theta)$ and an endomorphism $\eta$ of $A$ defined over $k=Q(\sqrt{-11})$ corresponding to $\chi$. Let $\mathfrak{D}_{k}$ denote the ring of all integers in $k$. In the present case, we see $\mathfrak{b}=\mathfrak{b}_{0}$ $=\left(\sqrt{3-\alpha_{0}}\right)$ and $\mathfrak{c}=\left(3-\alpha_{0}\right)$, and the condition $(* *)$ is satisfied with, e.g., $e=136$. Hence we can define an $\mathfrak{o}_{K}$-module $\mathfrak{x}=\{t \in A \mid \theta(\mathfrak{b}) t=0\}$ and an $\mathfrak{D}_{F}$-module $\mathfrak{y}=\{t \in \mathfrak{x} \mid(\eta-\theta(e)) t=0\}$. Let $k(\mathfrak{y})$ be as in $\S 1$, then from the action of $\mathrm{Gal}(k(\mathfrak{h}) / k)$ on $\mathfrak{y}$, we obtain an injective homomorphism

$$
r^{\prime}: \operatorname{Gal}(k(\mathfrak{y}) / k) \rightarrow\left(\mathfrak{o}_{F} / \mathfrak{c}\right)^{\times} \simeq(Z / 199 Z)^{\times},
$$

and put $r(\mathfrak{a})=r^{\prime}\left(\left(\frac{k(\mathfrak{h}) / k}{\mathfrak{a}}\right)\right)$.

Proposition 4.2. The field $k(y)$ is a ray class field over $k$ of conductor 11\} with a prime factor $\mathfrak{l}$ of 199 in $k$, and one has

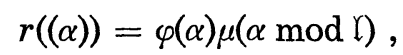

for every $\alpha$ in $k$ prime to $11 \mathfrak{l}$, where $\mu$ is the isomorphism of $\mathrm{D}_{k} / \mathfrak{l}$ onto $\mathfrak{b}_{F} /\left(3-\alpha_{0}\right)$ and $\varphi$ is a homomorphism of $\left(\mathfrak{o}_{k} /(11)\right)^{\times}$into $\left(\mathfrak{o}_{F} /\left(3-\alpha_{0}\right)\right)^{\times}$of order 22 such that

$$
\varphi(m)=\chi(m)
$$

for $m \in Z$.

Proof. Since every prime factor of the conductor $\mathfrak{f}$ of $k(\mathfrak{y}) / k$ divide $N(\mathfrak{l}) N=199 \cdot 11^{3}$ (see Shimura [12, §7.5, p. 181 and Prop. 7.23]), we may put $f=\prod_{\mathfrak{p}} \mathfrak{p}^{f_{\mathfrak{p}}}$, where $\mathfrak{p}$ runs through all prime factors of $199 \cdot 11$. By the same argument as in the proof of [14, Th. 2.3] we first obtain

$$
r((m))=\chi(m) \mu(m \bmod l)
$$


for every $m \in Z$ prime to $199 \cdot 11$. So we have $[k(\mathfrak{y}): k]=99$ or 198 , and the $\mathfrak{l}$-component $f_{\mathfrak{l}}=1$ by [14, Lemma 7.32]. To determine $f_{\mathrm{I} \varepsilon}$, we use the following fact which is nothing but [14, Th. 2.8] for the present case.

Let $l$ be a rational prime which divides $N(\mathrm{c})$ but not $N=11^{3}$. Suppose that $\chi(l)=1$, and $a_{l}$ is prme to $\mathfrak{c}=\left(3-\alpha_{0}\right)$. Then $\mathfrak{f}$ is divisible by only one of the prime factors of $l$ in $k$. Moreover if $\downarrow$ denotes the factor of $l$ which divides $\mathfrak{f}$, then

$$
r\left(\mathfrak{l}^{\mathfrak{c}}\right) \equiv a_{\imath} \bmod \mathfrak{c} .
$$

Take $l=199$. Since $a_{199}$ is prime to 199 , one has $f_{\mathrm{I} \varepsilon}=0$. Hence $\mathfrak{f}=(\sqrt{-11})^{m} \mathfrak{l}$ for some positive integer $m$. Owing to Hasse's conductor ramification theorem, we have $1 \leq m \leq 2$. Thus we have

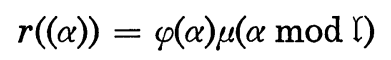

for every $\alpha$ in $k$ prime to 11 with a homomorphism $\varphi$ of $\left(o_{k} /(11)\right)^{\times}$into $\left(\mathfrak{o}_{F} / \mathfrak{c}\right)^{\times}$and the isomorphism $\mu$ of $\mathfrak{o}_{k} / \mathfrak{l}$ onto $\mathfrak{o}_{F} / \mathfrak{c}$. The order of $\varphi$ is determined as follows. Because $r\left(l^{\varepsilon}\right) \equiv a_{199} \equiv 133 \bmod \left(3-\alpha_{0}\right)$, we have $\varphi\left(l^{s}\right) \equiv 136$ $(\bmod 199)$. Therefore the order of $\varphi$ is 22 . Hence $m=2$ and $f=11$. This completes the proof of our proposition.

Remark 4.3. Let $p(\neq 199)$ be a rational prime such that $\chi(p)=1$, then $p$ decomposes into two distinct primes $\mathfrak{p}=(\gamma)$ and $\mathfrak{p}^{\varepsilon}=\left(\gamma^{c}\right)$ in $k$ $=Q(\sqrt{-11}) . \quad \gamma$ can be so chosen as $p=\gamma \gamma^{s}, \chi\left(\gamma+\gamma^{s}\right)=1$. Observe that $\varphi\left(\gamma^{11}\right)=\chi\left(-\gamma-\gamma^{\varepsilon}\right)=-1$. Let $\pi$ and $\pi^{\prime}$ be the solutions of $X^{2}-a_{p} X+p$ $\equiv 0 \bmod \left(3-\alpha_{0}\right)$. Then by [14, Th. 2.3], we have

$$
\pi^{11}+\pi^{\prime 11} \equiv-\gamma^{11}-\left(\gamma^{s}\right)^{11} \bmod \left(3-\alpha_{0}\right)
$$

Remark 4.4. Let $\alpha_{0}=e^{2 \pi i / 11}+e^{-2 \pi i / 11}$ as above. We note $\alpha_{0}$ is one of the fundamental units of $F_{11}$, and we have $N_{F_{11} / Q}\left(\alpha_{0}^{11}-1\right)=23^{2} \cdot 67 \cdot 199$. As was indicated in [4] for the case of $q=7^{3}$, the prime $N(\mathfrak{c})=199$ seems to be closely related to the factor 199 of $N_{F_{11} / Q}\left(\alpha_{0}^{11}-1\right)$.

Let us consider the endomorphism algebra $\operatorname{End}_{Q}(A)$. Put $\delta=\sqrt{3}-\alpha_{0}$, then $\theta(\delta)$ and $\eta$ generate a quaternion subalgebra $\mathfrak{A}$ of $\operatorname{End}_{\boldsymbol{Q}}(A)$ over $F=F_{11}$. Since $\delta^{2}=\left(3-\alpha_{0}\right) \in N_{F(\sqrt{-11}) / F}(F(\sqrt{-11})), \mathfrak{U}$ is isomorphic to $M_{2}(F)$. Denote by ' the canonical involution of $\mathfrak{A}$. Then we can find an element $\xi=a+b \theta(\delta)+c \eta+d \theta(\delta) \eta$ in $\mathfrak{U}$ with $a, b, c, d \in \theta\left(\mathfrak{o}_{F}\right) \cap$ End $(A)$ such that $\xi \xi^{\prime}=0$ and $\xi^{2}=e$ with $e \in \theta\left(\mathfrak{o}_{F}\right) \cap$ End $(A)$. Define an abelian subvariety 
$B$ of $A$ by

$$
\xi B=A
$$

Then $B$ is rational over $k, A=B+B^{\varepsilon}$ and $B^{\varepsilon}=\xi^{\varepsilon} A$. We can easily verify that $B \cap B^{s}$ is a finite group annihilated by $(e-2 b \theta(\delta))$. Denote by $\theta_{F}(a)$ the restriction of $\theta(a)$ to $B$ for $a \in F$, then we have an isomorphism $\theta_{F}$ of $F$ into $\operatorname{End}_{Q}(B)$.

Proposition 4.5. The notation being as above, the abelian variety $B$ is simple and $\operatorname{End}_{Q}(B)=\theta_{F}(F)$.

Proof. Let $\varphi_{p}$ be the $p$-th power Frobenius endomorphism of $B$ modulo $\mathfrak{p}$, where $\mathfrak{p}$ is a prime ideal in $k$ such that $N \mathfrak{p}=p$. By the table, we know that $F\left(\varphi_{3}\right) \neq F\left(\varphi_{5}\right)$. Therefore by the same argument as that of [12, Th. 7.39], we obtain our proposition.

\section{§. The case $q^{\nu}=19^{2}$}

In this section, we shall study particularly the case $\nu=2$, and give an example for $q^{\nu}=19^{2}$. Let $\psi$ be a character modulo $q$. Then for $f$ $=\sum_{n=1}^{\infty} a_{n} e^{2 \pi i n z} \in S_{\kappa}\left(\Gamma_{0}(q), \psi^{2}\right)$, put $f_{\bar{\psi}}=\sum_{n=1}^{\infty} a_{n} \bar{\psi}(n) e^{2 \pi i n z}$, then by [13, Prop. 3.64], we know $f_{\psi} \in S_{k}\left(\Gamma_{0}\left(q^{2}\right)\right)$. Put $S_{k}\left(\Gamma_{0}(q), \psi^{2}\right)^{\bar{\psi}}=\left\{f_{\bar{\psi}} \mid f \in S_{k}\left(\Gamma_{0}(q), \psi^{2}\right)\right\}$, $S_{\kappa}\left(S L_{2}(Z)\right)^{x}=\left\{f_{x} \mid f \in S_{\kappa}\left(S L_{2}(Z)\right)\right\}$ and $S_{k}^{0}\left(\Gamma_{0}(q)\right)^{x}=\left\{f_{x} \mid f \in S_{k}^{0}\left(\Gamma_{0}(q)\right)\right\}$. Then by [8, Prop. 7.1 and Prop. 7.2], we know $S_{\star}^{0}\left(\Gamma_{0}\left(q^{2}\right)\right)$ contains a subspace isomorphic to $\left(\oplus_{\{\psi\}, \psi^{2} \neq 1} S_{x}\left(\Gamma_{0}(q), \psi^{2}\right)\right) \oplus S_{k}^{0}\left(\Gamma_{0}(q)\right)^{x} \oplus S_{x}\left(S L_{2}(Z)\right)^{x}$, where the sum $\oplus_{\{\psi\}, \psi^{2} \neq 1}$ runs over a set of representatives of the pairs $\{\psi, \bar{\psi}\}, \psi^{2} \neq 1$, of the characters of $(Z / q Z)^{\times}$. Let $S_{k}^{n}\left(\Gamma_{0}\left(q^{2}\right)\right)$ denote the orthogonal complement of $S_{x}^{0}\left(\Gamma_{0}(q)\right)^{x} \oplus S_{k}\left(S L_{2}(Z)\right)^{x}$ in $S_{k}^{0}\left(\Gamma_{0}\left(q^{2}\right)\right)$ with respect to the Petersson inner product. Then $S_{k}^{n}\left(\Gamma_{0}\left(q^{2}\right)\right)$ is closed under the action of $W, \delta_{x}$ and Hecke operators $T_{n}$. Hence as in the case $\nu \geq 3$, we can define the subspaces $S_{\mathrm{I}}, S_{\mathrm{II}}, S_{\mathrm{II}}, S_{\mathrm{III}}$ satisfying $S_{k}^{n}\left(\Gamma_{0}\left(q^{2}\right)\right)=S_{\mathrm{I}} \oplus S_{\mathrm{II}} \oplus S_{\mathrm{II}_{x}} \oplus S_{\mathrm{III}}$ by

$$
\begin{aligned}
S_{\mathrm{I}} & =\left\{f \in S_{x}^{n}\left(\Gamma_{0}\left(q^{2}\right)\right)|f| W=f, f\left|\delta_{z} W=f\right| \delta_{x}\right\} \\
S_{\mathrm{II}} & =\left\{f \in S_{x}^{n}\left(\Gamma_{0}\left(q^{2}\right)\right)|f| W==f, f\left|\delta_{x} W=-f\right| \delta_{z}\right\} \\
S_{\mathrm{II}} & =\left\{f \in S_{x}^{n}\left(\Gamma_{0}\left(q^{2}\right)\right)|f| W=-f, f\left|\delta_{\chi} W=f\right| \delta_{\chi}\right\} \\
S_{\mathrm{III}} & =\left\{f \in S_{x}^{n}\left(\Gamma_{0}\left(q^{2}\right)\right)|f| W=-f, f\left|\delta_{\chi} W=-f\right| \delta_{x}\right\} .
\end{aligned}
$$

By the following lemma we know to which subspace $f_{\bar{\psi}}, \psi^{2} \neq 1$, belongs. 
LEMMA 5.1. Let $\psi$ be a character modulo $q$ and $f$ be an element of $S_{*}\left(\Gamma_{0}(q), \psi^{2}\right)$. Then one has

$$
f_{\bar{\psi}} \mid W=\psi(-1) f_{\bar{\psi}} .
$$

Proof. For $u \in Z$, put $\alpha_{u}=\left(\begin{array}{cc}1 & u / q \\ 0 & 1\end{array}\right)$, then we have

$$
f_{\Psi}=\frac{1}{W(\psi)} \sum_{u=1}^{q-1} \psi(u) f \mid\left[\alpha_{u}\right]_{\kappa}
$$

where $W(\psi)$ is the Gaussian sum of $\psi$. Hence we have

$$
f_{\psi}\left|W=\frac{1}{W(\psi)} \sum_{u=1}^{q-1} \psi(u) f\right|\left[\alpha_{u}\left(\begin{array}{rr}
0 & -1 \\
q^{2} & 0
\end{array}\right)\right]_{\kappa} .
$$

Since $(u, q)=1$, there exist $c, d \in Z$ such that $u d-q c=1$. Then we see

$$
\alpha_{u}\left(\begin{array}{rr}
0 & -1 \\
q^{2} & 0
\end{array}\right)=\left(\begin{array}{rr}
0 & -1 \\
q & 0
\end{array}\right)\left(\begin{array}{cr}
d & -1 \\
-q c & u
\end{array}\right)\left(\begin{array}{rr}
0 & -1 \\
q & 0
\end{array}\right)\left(\begin{array}{rr}
-1 & d / q \\
0 & -1
\end{array}\right)
$$

and $f\left[\left[\alpha_{u}\left(\begin{array}{lr}0 & -1 \\ q^{2} & 0\end{array}\right)\right]_{x}=\bar{\psi}^{2}(u) f \mid\left[\begin{array}{rr}-1 & d / q \\ 0 & -1\end{array}\right)\right]_{x}$. Therefore we obtain

$$
\begin{aligned}
f_{\bar{\psi}} \mid W & =\frac{1}{W(\psi)} \sum_{d=1}^{q-1} \psi(u) \bar{\psi}^{2}(u) f \mid\left[\left(\begin{array}{rr}
-1 & d / q \\
0 & -1
\end{array}\right)\right]_{x} \\
& =\frac{1}{W(\psi)} \sum_{d=1}^{q-1} \bar{\psi}(-u) f\left[\left(\begin{array}{cc}
1 & d / q \\
0 & 1
\end{array}\right)\right]_{k} \\
& =\psi(-1) f_{\Psi} .
\end{aligned}
$$

This completes the proof.

To calculate $\operatorname{tr} T_{n}$ on each subspace $S_{\mathrm{I}}, S_{\mathrm{II}}$, etc., we need the following lemma.

LEMMA 5.2. Let $n$ be a positive integer prime to $q$.

i) Put $S(1)=S_{\kappa}\left(S L_{2}(Z)\right) \oplus S_{\kappa}\left(S L_{2}(Z)\right)^{q} \oplus S_{\kappa}\left(S L_{2}(Z)\right)^{q^{2}} \oplus S_{\kappa}\left(S L_{2}(Z)\right)^{x}$, where $S_{\kappa}\left(S L_{2}(Z)\right)^{m}=\left\{f(m z) \mid f \in S_{\kappa}\left(S L_{2}(Z)\right)\right\}$ for a positive integer $m$. Then one has

$$
\begin{aligned}
& \operatorname{tr} \delta_{\chi} W \delta_{\chi} T_{n}\left|S(1)=\left(\chi(-1)+q^{-1}\right) \operatorname{tr} T_{n}\right| S_{x}\left(S L_{2}(Z)\right) \\
& \operatorname{tr} \delta_{z} W \delta_{\chi} W T_{n}\left|S(1)=2 q^{-1} \chi(-1) \operatorname{tr} T_{n}\right| S_{x}\left(S L_{2}(Z)\right) .
\end{aligned}
$$

ii) Put $S(q)=S_{k}^{0}\left(\Gamma_{0}(q)\right) \oplus S_{k}^{0}\left(\Gamma_{0}(q)\right)^{q} \oplus S_{*}^{0}\left(\Gamma_{0}(q)\right)^{x}$, where $S_{*}^{0}\left(\Gamma_{0}(q)\right)^{q}=\{f(q z) \mid$ $\left.f \in S_{*}^{0}\left(\Gamma_{0}(q)\right)\right\}$. Then one has 


$$
\begin{aligned}
& \operatorname{tr} \delta_{\chi} W \delta_{\chi} T_{n}\left|S(q)=\left(\chi(-1)+q^{-1}\right) \operatorname{tr} T_{n}\right| S_{x}^{0}\left(\Gamma_{0}(q)\right) \\
& \operatorname{tr} \delta_{\chi} W \delta_{\chi} W T_{n}\left|S(q)=2 q^{-1} \chi(-1) \operatorname{tr} T_{n}\right| S_{x}^{0}\left(\Gamma_{0}(q)\right)
\end{aligned}
$$

Proof. We prove only the formula for $\operatorname{tr} \delta_{z} W \delta_{x} T_{n}$ in the case of ii). The other cases can be treated in the same way. If $f$ is contained in $S_{*}^{0}\left(\Gamma_{0}(q)\right)^{q}$, obviously $f \mid \delta_{z} W \delta_{z} T_{n}=0$. Let $f$ be a primitive form in $S_{*}^{0}\left(\Gamma_{0}(q)\right)$, then we have by Lemma 5.1

$$
f\left|\delta_{\chi} W \delta_{\chi}=\chi(-1) f_{\chi}\right| \delta_{\chi}=\chi(-1)\left(f(z)-a_{q} f(q z)\right) .
$$

Let $f=g_{x}$ for a primitive form $g(z)$ in $S_{x}^{0}\left(\Gamma_{0}(q)\right)$, and $b_{q}$ be the $q$-th Fourier coefficient of $g$. Put $g \mid\left[\left(\begin{array}{rr}0 & -1 \\ q & 0\end{array}\right)\right]_{x}=\varepsilon g$, then we have

$$
\begin{aligned}
f \mid \delta_{\chi} W \delta_{\chi} & =\left(g(z)-b_{q} g(q z)\right) \mid W \delta_{\chi}=\left(\varepsilon q^{\kappa / 2} g(q z)-b_{q} \varepsilon q^{-\kappa / 2} g(z)\right) \\
& =q^{-1} f
\end{aligned}
$$

since $\varepsilon=-\bar{b}_{q} q^{1-k / 2}$. Our assertion follows from (5.1) and (5.2) easily.

By virtue of this lemma, we can express $\operatorname{tr} T_{n}$ on each space $S_{\mathrm{I}}, S_{\mathrm{II}}$, etc. by using formulae for $\operatorname{tr} T_{n}, \operatorname{tr} W T_{n}, \operatorname{tr} \delta_{\chi} W \delta_{\chi} T_{n}$, and $\operatorname{tr} \delta_{z} W \delta_{\chi} W T_{n}$. For example, on $S_{1}$, we have

$$
\begin{aligned}
\operatorname{tr} T_{n} \mid S_{\mathrm{I}}= & \frac{1}{4}\left\{\operatorname{tr} T_{n}\left|S_{\kappa}^{0}\left(\Gamma_{0}\left(q^{2}\right)\right)+\operatorname{tr} W T_{n}\right| S_{k}^{0}\left(\Gamma_{0}\left(q^{2}\right)\right)\right. \\
& +\operatorname{tr} \delta_{\chi} W \delta_{\chi} T_{n}\left|S_{k}\left(\Gamma_{0}\left(q^{2}\right)\right)+\operatorname{tr} \delta_{\chi} W \delta_{x} W T_{n}\right| S_{\kappa}\left(\Gamma_{0}\left(q^{2}\right)\right) \\
& -\left(\chi(-1)+q^{-1}+2 \chi(-1) q^{-1}\right)\left(\operatorname{tr} T_{n} \mid S_{\kappa}\left(S L_{2}(Z)\right) \oplus S_{\kappa}^{0}\left(\Gamma_{0}(q)\right)\right\}
\end{aligned}
$$

Now let us discuss a numerical example in the case where $q=19^{2}$ and $\kappa=2$. We find $\operatorname{dim} S_{2}^{0}\left(\Gamma_{0}\left(19^{2}\right)\right)=20$ and $\operatorname{dim} S_{2}^{0}\left(\Gamma_{0}(19)\right)^{x}=1$. In the following we give the characteristic polynomials of Hecke operators $T_{n}$ acting on $S_{\mathrm{I}}$ and $S_{\mathrm{III}}$ for several $n$. We note $S_{\mathrm{II}}=\bigoplus_{\{\psi\}, \psi^{2} \neq 1, \psi(-1)=1} S_{2}\left(\Gamma_{0}(19)\right.$, $\left.\psi^{2}\right)^{\bar{\psi}}$ and $S_{\mathrm{II}_{\chi}}=\bigoplus_{\{\psi\}, \psi^{2} \neq 1, \psi(-1)=-1} S_{2}\left(\Gamma_{0}(19), \psi^{2}\right)^{\bar{\psi}}$.

\begin{tabular}{c|c|c|c|c|c|c|c}
\multirow{2}{*}{$n$} & \multirow{2}{*}{$\chi(n)$} & \multicolumn{5}{|c}{ characteristic polynomial of $T_{n}$} \\
\cline { 3 - 7 } & & \multicolumn{3}{|c|}{$S_{\text {I }}$} & \multicolumn{4}{|c}{$S_{\text {III }}$} \\
\hline 2 & -1 & $X$ & $X^{4}-5 X^{2}+5$ & $X^{2}-5$ & $X^{2}-5$ & $X^{2}+X-1$ & $X^{2}-X-1$ \\
5 & +1 & $X+1$ & $\left(X^{2}+2 X-4\right)^{2}$ & $X^{2}-X-1$ & $X^{2}-X-1$ & $X^{2}-2 X-4$ & $X^{2}-2 X-4$ \\
7 & +1 & $X-3$ & $\left(X^{2}+4 X-1\right)^{2}$ & $X^{2}+2 X-4$ & $X^{2}+2 X-4$ & $(X-3)^{2}$ & $(X-3)^{2}$
\end{tabular}

The one-dimensional part in $S_{\mathrm{I}}$ is obtained by a primitive form corresponding to an $L$-function of the field $Q(\sqrt{-19})$. 
Let $f=\sum_{n=1}^{\infty} a_{n} e^{2 \pi i n z}$ be a primitive form in the four-dimensional part of $S_{\mathrm{I}}$. We may assume

$$
a_{2}=\sqrt{\frac{5+\sqrt{5}}{2}}, \quad a_{5}=-1-\sqrt{5}, \quad a_{7}=-2+\sqrt{5} .
$$

(These coefficients are obtained from $\operatorname{tr} T_{n}$ for several $n$, which are not given here.) Let $K$ be the field generated over $\boldsymbol{Q}$ by $a_{n}$ for all $n$, then $K=Q\left(\sqrt{\frac{5+\sqrt{5}}{2}}\right)$, and we see $f$ and $K$ satisfy the condition $(*)$ in $\S 1$. In this case we have $F=\boldsymbol{Q}(\sqrt{5)}, \mathfrak{c}=(\sqrt{5})$, and $k=\boldsymbol{Q}(\sqrt{-19)}$ in the notation of $\S 1$ and $\S 4$. Since the condition $(* *)$ is satisfied by taking $e=1$, we can consider the $\mathfrak{o}_{F}$-module $\mathfrak{y}$, the extension $k(\mathfrak{y}) / k$, and an injective homomorphism

$$
r^{\prime}: \operatorname{Gal}(k(\mathfrak{h}) / k) \rightarrow\left(\mathfrak{o}_{F} / \mathfrak{c}\right)^{\times} \simeq(Z / 5 Z)^{\times} .
$$

Put $r(\mathfrak{a})=r^{\prime}\left(\left(\frac{k(\mathfrak{h}) / k}{\mathfrak{a}}\right)\right)$.

Proposition 5.3. The field $k(\mathfrak{y})$ is a ray class field of conductor $(\sqrt{-19}) \mathfrak{l}$ with a prime factor $\mathfrak{l}$ of 5 , and one has

$$
r((\alpha))=\varphi(\alpha) \mu(\alpha \bmod l)
$$

for every $\alpha$ in $k$ prime to 191 , where $\mu$ is the isomorphism of $\mathfrak{o}_{k} / l$ onto $\mathfrak{o}_{F} / \mathfrak{c}$ and $\varphi$ is a homomorphism of $\left(\mathfrak{o}_{k} /(\sqrt{-19})\right)^{\times}$into $\left(\mathrm{o}_{F} / \mathrm{c}\right)^{\times}$of order 2.

This proposition can be proved by virtue of (5.3) in the same way as Prop. 4.2, and we omit the proof. We can show also in this case that there exists a simple abelian subvariety $B$ defined over $k$ of the abelian variety $A$ associated with $f$ such that $A=B+B^{\varepsilon}$ and $\operatorname{End}_{Q}(B)=Q(\sqrt{5})$, where $\varepsilon$ is the generator of $\mathrm{Gal}(k / Q)$.

\section{§6. Formulae for $\operatorname{tr} \delta_{x}$ and $\operatorname{tr} T_{q} W$}

For a character $\psi$ modulo $q^{\nu}$ and a positive integer $\kappa \geq 2$, let $S_{k}\left(\Gamma_{0}\left(q^{\nu}\right), \psi\right)$ be as in $\S 1$. We assume $\psi(-1)=(-1)^{k}$ and the conductor $\mathfrak{f}_{\psi}$ satisfies $\mathfrak{f}_{\psi} \leq q^{\nu-1}$. If $\nu \geq 2$, we can define the twisting operator $\delta_{z}$ on $S_{k}\left(\Gamma_{0}\left(q^{\nu}\right), \psi\right)$ as in $\S 1$. Let us express $\delta_{z}$ as an action of double cosets. We use the same notation as in $\S 2$. Put $\Xi_{q}=U_{q}\left(\begin{array}{ll}q & 1 \\ 0 & q\end{array}\right) U_{q}$, then we see 


$$
\Xi_{q}=\left(\begin{array}{cc}
q Z_{q}^{\times} & Z_{q}^{\times} \\
q^{\nu+1} Z_{q} & q Z_{q}^{\times}
\end{array}\right)
$$

For $g=\left(\begin{array}{ll}a & b \\ c & d\end{array}\right) \in \Xi_{q}$, put

$$
\lambda(g)=(\psi \chi)^{-1}(a / q) \chi(b),
$$

and for $g \in \prod_{p \neq q} U_{p} \times \Xi_{q} \times U_{\infty}$, put

$$
\lambda(g)=\lambda\left(g_{q}\right),
$$

where $g_{q}$ denotes the $q$-component of $g$. For $\gamma=\left(\begin{array}{ll}a & b \\ c & d\end{array}\right) \in \Gamma_{0}\left(q^{\nu}\right)$, put $\psi(\gamma)$ $=\psi(a)^{-1}$. Then for $g \in\left(\prod_{p \neq q} U_{p} \times E_{q} \times U_{\infty}\right) \cap G L_{2}(Q)$, we have

$$
\lambda\left(\gamma_{1} g \gamma_{2}\right)=\psi\left(\gamma_{1}\right) \lambda(g) \psi\left(\gamma_{2}\right) .
$$

Let $\left(\prod_{p \neq q} U_{p} \times \Xi_{q} \times U_{\infty}\right) \cap G L_{2}(\boldsymbol{Q})=\bigcup_{u=1}^{d} \Gamma_{0}\left(q^{\nu}\right) \alpha_{u}$ be a disjoint union. Then by Lemma 2.1 and (6.1), we obtain

$$
f\left|\delta_{\chi}=\frac{1}{W(\chi)} \sum_{u=1}^{d} \lambda\left(\alpha_{u}\right)^{-1} f\right|\left[\alpha_{u}\right]_{\kappa} .
$$

Now in the case where $\nu=1$ and $\psi=\chi$, we consider the operator $T_{q} W$. Put

$$
\Xi_{q}=\left\{g \in\left(\begin{array}{ll}
q Z_{q} & Z_{q}^{\times} \\
q^{2} Z_{q} & q Z_{q}
\end{array}\right) \mid v_{q}(\operatorname{det} g)=2\right\}
$$

For $g=\left(\begin{array}{ll}a & b \\ c & d\end{array}\right) \in \Xi_{q}$, define

$$
\lambda(g)=\chi(b),
$$

and for $g \in \prod_{p \neq q} U_{p} \times \Xi_{q} \times U_{\infty}$, put

$$
\lambda(g)=\lambda\left(g_{q}\right),
$$

where $g_{q}$ denotes the $q$-component of $g$. Then we have

$$
\lambda\left(\gamma_{1} g \gamma_{2}\right)=\chi\left(\gamma_{1}\right) \lambda(g) \chi\left(\gamma_{2}\right) \quad \text { for } \gamma_{1}, \gamma_{2} \in \Gamma_{0}(q)
$$

where $\chi(\gamma)=\chi(a)$ for $\gamma=\left(\begin{array}{ll}a & b \\ c & d\end{array}\right) \in \Gamma_{0}(q)$. Let $\left(\prod_{p \neq q} U_{p} \times \Xi_{q} \times U_{\infty}\right) \cap G L_{2}(\boldsymbol{Q})$ $=\bigcup_{u=1}^{d} \Gamma_{0}(q) \alpha_{u}$ be a disjoint union, then we can verify for $f \in S_{\kappa}\left(\Gamma_{0}(q), \chi\right)$ 


$$
f\left|T_{q} W=q^{k / 2-1} \sum_{u=1}^{d} \lambda\left(\alpha_{u}\right)^{-1} f\right|\left[\alpha_{u}\right]_{\kappa} .
$$

THEOREM 6.1. Let $\psi$ be a character modulo $q^{\nu}$ with the conductor $f_{\psi}$ such that $\mathrm{f}_{\psi} \leq q^{\nu-1}$, and $\kappa$ be a positive integer such that $\kappa \geq 2$. Assume $q \geq 5$ and $\psi(-1)=(-1)^{x}$. If $\nu \geq 2$, one has

$$
\operatorname{tr} \delta_{x} \mid S_{x}\left(\Gamma_{0}\left(q^{\nu}\right), \psi\right)= \begin{cases}q^{\nu-1-[\nu / 2]} \frac{1-\chi(-1)}{2} h(-q), & \text { if } f_{\psi} \leq q^{[\nu / 2]} \\ 0, & \text { otherwise, }\end{cases}
$$

and if $\nu=1$, one has

$$
\operatorname{tr} T_{q} W \mid S_{\varepsilon}\left(\Gamma_{0}(q), \chi\right)= \begin{cases}\chi(-1) W(\chi) q^{k / 2-1} h(-q), & q \equiv 3(\bmod 4) \\ 0, & q \equiv 1(\bmod 4),\end{cases}
$$

where $h(-q)$ is the class number of $\mathbf{Q}(\sqrt{-q})$.

Proof. Put $T=\operatorname{tr} \delta_{x} \mid S_{x}\left(\Gamma_{0}\left(q^{\nu}\right), \psi\right)$ if $\nu \geq 2$, and $T=\frac{1}{\chi(-1) W(\chi)} q^{\overline{s / 2-1}}$ $\operatorname{tr} T_{q} W \mid S_{\kappa}\left(\Gamma_{0}(q), \chi\right)$ if $\nu \geq 1$. Let $\Xi_{A}$ denote the set $\prod_{p \neq q} U_{p} \times \Xi_{q} \times U_{\infty}$ and put $\Xi=\Xi_{A} \cap G L_{2}(\boldsymbol{Q})$. We note $\Xi$ contains neither scalars nor hyperbolic elements whose characteristic roots are contained in $\boldsymbol{Q}$. By EichlerSelberg's trace formula, we have $T=t_{e}+t_{p}$, where

$$
\begin{aligned}
t_{e} & =-\frac{1}{W(\chi)} \sum_{\alpha \in g_{e} / \sigma_{0}\left(q^{\nu}\right)} \frac{\lambda(\alpha)^{-1}}{[\Gamma(\alpha):\{ \pm 1\}]} \frac{\eta_{\alpha}^{\kappa-1}-\zeta_{\alpha}^{\kappa-1}}{\eta_{\alpha}-\zeta_{\alpha}} q^{-(\kappa-2)} \\
t_{p} & =\frac{1}{W(\chi)} \lim _{s \rightarrow 0}\left(\frac{1}{2 \pi}\right) \sum_{\alpha \in B_{p} / \overbrace{0}\left(q^{\nu}\right)} \lambda(\alpha)^{-1} \frac{\operatorname{sgn}(\alpha)^{k}}{2}\left(\frac{i}{m(\alpha)}\right)^{1+s} .
\end{aligned}
$$

Here $\Xi_{e}$ (resp. $\Xi_{p}$ ) denotes the set of all elliptic elements (resp. parabolic elements fixing cusps of $\left.\Gamma_{0}\left(q^{\nu}\right)\right)$ in $\Xi$. $\zeta_{\alpha}$ and $\eta_{\alpha}$ are the characteristic roots of $\alpha$ determined by $\rho \alpha \rho^{-1}=\left(\begin{array}{cc}\eta_{\alpha} & 0 \\ 0 & \zeta_{\alpha}\end{array}\right)$, where $\rho=\left(\begin{array}{ll}1 & -z_{0} \\ 1 & -\bar{z}_{0}\end{array}\right)$ for the fixed point $z_{0}$ of $\alpha$ in $\mathfrak{S}$. For a parabolic element $\alpha, m(\alpha)$ has the same meaning as in (2.4), and $\operatorname{sgn}(\alpha)=\operatorname{sgn} \zeta_{\alpha}$ for the characteristic root $\zeta_{\alpha}$ of $\alpha$. First we show $t_{e}=0$. Let $f(X)$ be the characteristic polynomial of an elliptic element in $\varepsilon$, then $f(X)=X^{2}+q^{2}$, or $X^{2} \pm q X+q^{2}$. For a $Z$-order $A$ of $K(f)$, we see by the theorem of Hijikata quoted in $\S 2$ that if $C_{A}(\alpha, \Lambda) \cap E_{A}$ is not empty, then $\Lambda_{q}=Z_{q}[X] /(f(X))$. Put $U_{q}^{+}=\left\{g \in U_{q}\right.$ $\mid \chi(\operatorname{det} g)=1\}$ and $U^{+}=\prod_{p \neq q} U_{p} \times U_{q}^{+} \times U_{\infty}$. Then $U^{+}$is a subgroup of 
$U$ of index 2 and for $\gamma \in U^{+}$and $g \in \Xi_{A}$, we have

$$
\lambda\left(\gamma^{-1} g \gamma\right)=\lambda(g)
$$

For a $Z$-order $\Lambda$ of $K(f)$, let $\theta$ be the map $\theta: C(\alpha, \Lambda) \cap E / \widetilde{\Gamma_{0}\left(q^{\nu}\right) \rightarrow C_{A}(\alpha, \Lambda)}$

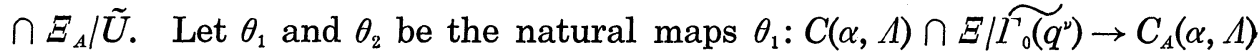
$\cap \Xi_{A} / \tilde{U}^{+}$, and $\theta_{2}: C_{A}(\alpha, \Lambda) \cap \Xi_{A} / \tilde{U}^{+} \rightarrow C_{A}(\alpha, \Lambda) \cap \Xi_{A} / \tilde{U}$ induced by the inclusion, then $\theta$ is the composite of $\theta_{1}$ and $\theta_{2}$. Let $N$ denote the norm map of $K(f)$ to $\boldsymbol{Q}$, and $\boldsymbol{Q}_{A}^{+}$denotes the subgroup $\left(\prod_{p \neq q} \boldsymbol{Z}_{p}^{\times} \times \boldsymbol{Z}_{q}^{+} \times \boldsymbol{R}_{+}^{\times}\right) \boldsymbol{Q}^{\times}$of $\boldsymbol{Q}_{A}^{\times}$, where $Z_{q}^{+}=\left\{x \in Z_{q} \mid \chi(x)=1\right\}$ and $\boldsymbol{R}_{+}^{\times}=\{x \in R \mid x>0\}$. Since $\Lambda_{q}=Z_{q}[X] /$ $(f(X))$ is not the maximal order of $K(f)_{q}$, we have $N\left(\prod_{p} \Lambda_{p}^{\times} \times K(f)_{\infty}^{\times}\right) \subset \boldsymbol{Q}_{A}^{+}$. Hence for a class $\tilde{g}$ of $C_{A}(\alpha, \Lambda) \cap \Xi_{A} / \tilde{U}, \theta_{2}^{-1}(\tilde{g})$ consists of two classes $\tilde{g}_{1}$ and $\tilde{g}_{2}$ of $C_{A}(\alpha, \Lambda) \cap \Xi_{A} / \tilde{U}^{+}$and $\lambda\left(\tilde{g}_{1}\right)=-\lambda\left(\tilde{g}_{2}\right)$. We claim $\left|\theta_{1}^{-1}\left(\tilde{g}_{1}\right)\right|=\left|\theta_{1}^{-1}\left(\tilde{g}_{2}\right)\right|$ $=\frac{1}{2} h(K(f), A)$. Our assertion on $t_{e}$ follows from this. Since we assume $q \geq 5, N\left(K(f)_{A}^{\times}\right) \boldsymbol{Q}_{A}^{+}=\boldsymbol{Q}_{A}^{\times}$, hence $\operatorname{det}\left(\boldsymbol{Q}[\alpha]_{A}^{\times}\right) \boldsymbol{Q}_{A}^{+}=\boldsymbol{Q}_{A}^{\times}$, where $\boldsymbol{Q}[\alpha]_{A}=\boldsymbol{Q}[\alpha]$ $\otimes_{\boldsymbol{Q}} \boldsymbol{Q}_{A}$. Therefore we have $G L_{2}\left(\boldsymbol{Q}_{A}\right)=\boldsymbol{Q}[\alpha]_{A}^{\times} G L_{2}(\boldsymbol{Q}) U^{+}$, so $\theta_{1}$ is surjective. Let $u_{0}$ be an element of $U$ such that $u_{0} \notin U^{+}$. Then we may assume $g_{1}$ $=h^{-1} \alpha h, g_{2}=u_{0}^{-1} h^{-1} \alpha h u_{0}$ for $h \in G L_{2}\left(\boldsymbol{Q}_{A}\right)$ and we see $\left|\theta_{1}^{-1}\left(\widetilde{g_{1}}\right)\right|=\left|\boldsymbol{Q}[\alpha]^{\times}\right|$ $\left(\boldsymbol{Q}[\alpha]_{A}^{\times} h U^{+}\right) \cap G L_{2}(\boldsymbol{Q}) / \Gamma_{0}\left(q^{\nu}\right) \mid$ and $\left|\theta_{1}^{-1}\left(\widetilde{g_{2}}\right)\right|=\left|\boldsymbol{Q}[\alpha]^{\times} \backslash\left(\boldsymbol{Q}[\alpha]_{A}^{\times} h u_{0}\right) \cap G L_{2}(\boldsymbol{Q}) / \Gamma_{0}\left(q^{\nu}\right)\right|$. Put $G_{1}=\left\{x \in \boldsymbol{Q}[\alpha]_{A}^{\times} \mid \operatorname{det}(x h) \in \boldsymbol{Q}_{A}^{+}\right\}$, then $\left(\boldsymbol{Q}[\alpha]_{A}^{\times} h U^{+}\right) \cap G L_{2}(\boldsymbol{Q})=\left(G_{1} h U\right)$ $\cap G L_{2}(\boldsymbol{Q})$. By the same calculation as in Prop. 2.6, we obtain $\left|\theta_{1}^{-1}\left(\widetilde{g_{1}}\right)\right|$ $=\left|G_{1} / \boldsymbol{Q}[\alpha]^{\times}\left(h U h^{-1} \cap \boldsymbol{Q}[\alpha]_{A}\right)\right|=\frac{1}{2} h(K(f), \Lambda)$, and in the same way $\left|\theta_{1}^{-1}\left(\widetilde{g_{2}}\right)\right|$ $=\frac{1}{2} h(K(f), \Lambda)$. Now let us calculate $t_{p}$. Let $\alpha$ be an element of $\Xi_{p}$, then the characteristic polynomial $f_{\alpha}$ is either $X^{2}-2 q X+q^{2}$, or $X^{2}+2 q X+q^{2}$. Since the contribution from the parabolic elements $\alpha$ with $f_{\alpha}=X^{2}-2 q X$ $+q^{2}$ is the same with that from the parabolic elements $\alpha$ with $f_{\alpha}=X^{2}$ $+2 q X+q^{2}$, we may assume $f=X^{2}-2 q X+q^{2}$. Put $f(X)=X^{2}-2 q X$ $+q^{2}$, and $\Omega=\left\{x \in Z \mid f(x) \equiv 0\left(\bmod q^{\nu+1}\right)\right\}$ if $\nu \geq 2$, and $\Omega=\{q x \mid x \in Z,(x, q)=1\}$ if $\nu=1$. For $x \in \Omega$, put

$$
\alpha_{x}=\left(\begin{array}{cc}
x & 1 \\
-f(x) & 2 q-x
\end{array}\right)
$$

then $\alpha_{x}$ is contained in $C\left(\alpha, \Lambda_{0}\right) \cap \Xi$ for $\Lambda_{0}=Z[X] /(f(X))$. For $m \in Z$, put $\alpha_{x, m}=q+m\left(\alpha_{x}-q\right)$ and for a positive integer $m$, let $\Lambda(m)$ denote the $Z$ order of $K(f)$ such that $\left[\Lambda(m): \Lambda_{0}\right]=m$. Then we see easily that for a positive integer $m$ prime to $q,\left\{\alpha_{x, m} \mid x \in \Omega\left(\bmod q^{\nu}\right)\right\} \cup\left\{\alpha_{x,-m} \mid x \in \Omega\left(\bmod q^{\nu}\right)\right\}$

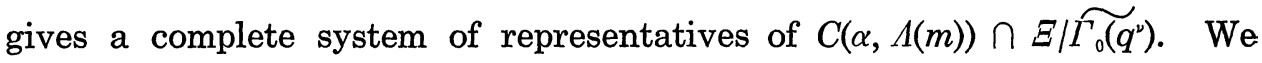


have $m\left(\alpha_{x, m}\right)=m / q$ and $\lambda\left(\alpha_{x, m}\right)=\psi(1+m(x-q) / q) \chi(m)$ for $m \in Z$, where we put $\psi=$ the identity character if $\nu=1$. Therefore we obtain

$$
\begin{aligned}
t_{p}= & \frac{1}{W(\chi)} \lim _{s \rightarrow 0} \frac{q i}{2 \pi} \sum_{\substack{m=1 \\
(m, q)=1}}^{\infty} \frac{1}{m^{1+s}}\left(\sum_{x \in \Omega\left(\bmod q^{\nu}\right)} \psi(1+m(x-q) / q) \chi(m) e^{\pi i s / 2}\right. \\
& \left.-\sum_{x \in \Omega\left(\bmod q^{\nu}\right)} \psi(1-m(x-q) / q) \chi(-m) e^{-\pi i s / 2}\right) .
\end{aligned}
$$

Since we have for $m \in Z$

$$
\sum_{x \in \Omega\left(\bmod q^{\nu}\right)} \psi(1+m(x-q) / q)= \begin{cases}\left|\Omega\left(\bmod q^{\nu}\right)\right|, & f_{\psi} \leq q^{[\nu / 2]} \\ 0, & \text { otherwise },\end{cases}
$$

and $\left|\Omega\left(\bmod q^{\nu}\right)\right|=q^{\nu-1-[\nu / 2]}$, we obtain

$$
\begin{aligned}
t_{p} & =\frac{1}{W(\chi)} \frac{q i}{2 \pi} q^{\nu-1-[\nu / 2]}(1-\chi(-1)) L(1, \chi) \\
& =q^{\nu-1-[\nu / 2]} \frac{1}{2}(1-\chi(-1)) h(-q) .
\end{aligned}
$$

This completes the proof.

Put $\mathfrak{q}=(\sqrt{-q})$. Let $\lambda$ be a Grössencharacter of $\boldsymbol{Q}(\sqrt{-q})$ satisfying

$$
\begin{gathered}
\lambda((\alpha))=\left(\frac{\alpha}{|\alpha|}\right)^{\varepsilon-1} \quad \text { for } \alpha \equiv 1 \bmod ^{\times} \mathfrak{q}^{\nu-1} \\
\lambda((n))=\psi(n) \chi(n) \quad \text { for } n \in Z .
\end{gathered}
$$

For $\lambda$, put $f_{\lambda}(z)=\sum_{a} \lambda(\mathfrak{a}) N(\mathfrak{a})^{(x-1) / 2} e^{2 \pi i N(a) z}$, then we know by [14] $f_{\lambda}$ is contained in $S_{k}\left(\Gamma_{0}\left(q^{\nu}\right), \psi\right)$. If $\nu \geq 2$, we see $f=f_{\lambda}$ satisfies

$$
\left(f \mid \delta_{\chi}\right)=f(z)+c f(q z) \quad \text { for some } c .
$$

If $\nu=1, f=f_{\lambda}$ satisfies

$$
f=f_{\rho},
$$

where $f_{\rho}=\sum_{n=1}^{\infty} \bar{a}_{n} e^{2 \pi i n z}$ for $f=\sum_{n=1}^{\infty} a_{n} e^{2 \pi i n z}$. On the other hand, we can prove

Corollary 6.2. Let $q$ and $\psi$ be as in Th 6.1. Let $M$ be the subspace of $S_{x}\left(\Gamma_{0}\left(q^{\nu}\right), \psi\right)$ spanned by the common eigen-functions of $T_{n}$ for all $n$ prime to $q$ satisfying (6.6) if $\nu \geq 2$ or (6.7) if $\nu=1$ and $\psi=\chi$. Then $M$ is spanned by $f_{\lambda}$ for all $\lambda$ satisfying (6.4) and (6.5).

Proof. If $\nu \geq 2$, it is easy to see $\operatorname{tr} \delta_{x} \mid S_{k}\left(\Gamma_{0}\left(q^{\nu}\right), \psi\right)=\operatorname{dim} M$. If $\nu=1$, 
by [1, Th. 2] for a primitive form $f$ in $S_{x}\left(\Gamma_{0}(q), \chi\right)$, we have

$$
f \mid T_{q} W=\chi(-1) W(\chi) q^{\kappa / 2-1} f_{\rho} .
$$

Hence we obtain $\frac{1}{\chi(-1) W(\chi) q^{\kappa / 2-1}} \operatorname{tr} T_{q} W \mid S_{\kappa}\left(\Gamma_{0}(q), \chi\right)=\operatorname{dim} M$. For $\lambda$ satisfying (6.4) and (6.5), $f_{\lambda}$ is contained in $M$ and we see there exist exactly $q^{\nu-1-[\nu / 2]} h(-q)$ such Grössencharacters if $\mathfrak{f}_{\psi} \leq q^{[\nu / 2]}$ and otherwise there are no such Grössencharacters. Since $f_{\lambda}$ 's are linearly independent over $C$ for such $\lambda$ 's, our assertion is proved.

Remark 6.3. The trace of the twisting operator has been given in Shimura [15] in the case where $\nu=2$ by another method. The special case of Cor. 6.3 was proved by Asai [2] as an application of Doi-Naganuma lifting for imaginary quadratic fields. In [9], Ribet proved a general result on the characterization of cusp forms associated with $L$-functions with Grössencharacters of imaginary quadratic fields. Cor. 6.3 gives another proof of the results of Asai and Ribet in the special cases.

\section{REFERENCES}

[1] T. Asai, On the Fourier coefficients of automorphic forms at various cusps and some applications to Rankin's convolution, J. Math. Soc. Japan, 28 (1976), 48-61.

[2] - On the Doi-Naganuma lifting associated with imaginary quadratic fields, Nagoya Math. J., 71 (1978), 149-167.

[ 3 ] A. O. L. Atkin and J. Lehner, Hecke operators on $\Gamma_{0}(m)$, Math. Ann., 185 (1970), 134-160.

[4] K. Doi and M. Yamauchi, On the Hecke operators for $\Gamma_{0}(N)$ and class fields over quadratic number fields, J. Math. Soc. Japan, 25 (1973), 629-643.

[5] M. Eichler, Eine Verallgemeinerung der Abelschen Integrale, Math. Z., 67 (1957), 267-298.

[6] H. Hijikata, Explicit formula of the traces of Hecke operators for $\Gamma_{0}(N)$, J. Math. Soc. Japan, 26 (1974), 56-82.

[ 7 ] H. Ishikawa, On the trace formula for Hecke operators, J. Fac. Sci. Univ. Tokyo, 21 (1974), 357-376.

[8] A. Pizer, Theta series and modular forms of level $p^{2} M$ (preprint).

[9] K. Ribet, Galois representations attached to eigenforms with Nebentypus, Proceedings International Conference, University of Bonn, 1976, Lecture Notes in Math., 601, Springer, 1977.

[10] H. Saito, On Eichler's trace formula, J. Math. Soc. Japan, 24 (1972), 333-340.

[11] H. Shimizu, On traces of Hecke operators, J. Fac. Sci. Univ. Tokyo, 10 (1963), 1-19.

[12] G. Shimura, Introduction to the arithmetic theory of automorphic functions, Publ. Math. Soc. Japan, No. 11, Iwanami Shoten and Princeton University Press, 1971.

[13] —, On elliptic curves with complex multiplication as factors of the jacobian of modular function fields, Nagoya Math. J., 43 (1971), 199-208. 
[14] G. Shimura, Class fields over real quadratic fields and Hecke operators, Ann. of Math., 95 (1972), 130-190.

[15] — - On the factors of the jacobian variety of a modular function field, J. Math. Soc. Japan, 25 (1973), 523-543.

[16] M. Yamauchi, On the traces of Hecke operators for a normalizer of $\Gamma_{0}(N)$, J. Math. Kyoto Univ., 13 (1973), 403-411.

Department of Mathematics College of General Education

Kyoto University 